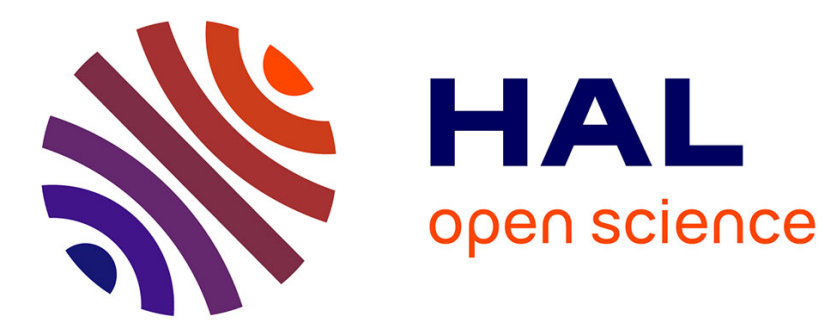

\title{
Discrete reconfiguration planning for Cable-Driven Parallel Robots
}

Lorenzo Gagliardini, Stéphane Caro, Marc Gouttefarde, Alexis Girin

\section{To cite this version:}

Lorenzo Gagliardini, Stéphane Caro, Marc Gouttefarde, Alexis Girin. Discrete reconfiguration planning for Cable-Driven Parallel Robots. Mechanism and Machine Theory, 2016, 100, pp.313-337. 10.1016/j.mechmachtheory.2016.02.014 . hal-01400440

\section{HAL Id: hal-01400440 \\ https://hal.science/hal-01400440}

Submitted on 22 Nov 2016

HAL is a multi-disciplinary open access archive for the deposit and dissemination of scientific research documents, whether they are published or not. The documents may come from teaching and research institutions in France or abroad, or from public or private research centers.
L'archive ouverte pluridisciplinaire $\mathbf{H A L}$, est destinée au dépôt et à la diffusion de documents scientifiques de niveau recherche, publiés ou non, émanant des établissements d'enseignement et de recherche français ou étrangers, des laboratoires publics ou privés. 


\title{
Discrete Reconfiguration Planning for Cable-Driven Parallel Robots
}

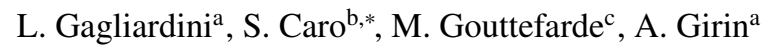 \\ a IRT Jules Verne, Bouguenais, 44340, France \\ ${ }^{b}$ IRCCyN-CNRS, 1, Rue de la Nö̈, 44321, Nantes Cedex 3, France \\ ${ }^{c}$ LIRMM-CNRS-UM, 161, Rue Ada, 34095, Montpellier Cedex 5, France
}

\begin{abstract}
Cable-Driven Parallel Robots (CDPRs) are a class of parallel robots whose legs consist of cables. In most previous studies, the positions of the cable connection points on the moving platform and on the base frame are fixed, these positions being determined during the CDPR design. However, such fixed-configuration CDPRs are not always suitable and some situations require reconfiguration capabilities, e.g. a cluttered environment where cable collisions with objects in the CDPR workspace cannot be completely avoided without reconfigurations. This paper deals with Reconfigurable Cable-Driven Parallel Robots (RCDPRs) whose cable connection points on the base frame can be positioned at a possibly large but discrete set of possible locations. Means to select and optimize the sequence of discrete reconfigurations allowing the RCDPR moving platform to follow a prescribed path are introduced. A so-called feasibility map is first generated. For each possible configuration of the RCDPR, this map stores the feasible or unfeasible character of each point of the discretized prescribed path, according to user-defined constraints which ensure a proper functioning of the RCDPR. The feasibility map is next analyzed in order to determine minimum sets of configurations which allow the RCDPR to follow the whole prescribed path. Finally, the corresponding discrete reconfiguration planning problem is represented as a graph whose nodes correspond to feasible RCDPR reconfigurations. The arcs of the graph are weighted by a user-defined cost function so that the graph can be searched for an optimal reconfiguration strategy using Dijkstras algorithm.
\end{abstract}

Keywords: Cable-Driven Parallel Robot, Reconfigurability, Reconfiguration Strategy, Graph Based Optimization

\footnotetext{
* Corresponding Author.

Email addresses: lorenzo.gagliardini@irt-jules-verne.fr (L. Gagliardini), Stephane.Caro@irccyn.ec-nantes.fr (S. Caro), marc.gouttefarde@lirmm.fr (M. Gouttefarde), alexis.girin@irt-jules-verne.fr (A. Girin)
} 


\section{Introduction}

Several industrial sectors, e.g. the naval and renewable energy industries, are facing the necessity to manufacture novel products of large dimensions and having complex shapes. In order to improve such manufacturing processes, the IRT Jules Verne promoted the investigation of new technologies. In this context, CAROCA ${ }^{1}$ project aims at investigating the performance of Cable Driven Parallel Robots (CDPRs) to manufacture large products in cluttered industrial environments [1]. CDPRs are a particular class of parallel robots whose moving platform is connected to the robot fixed base frame by a number of cables as illustrated in Fig. 1. Hereafter, the connection points between the cables and the base frame will be referred to as exit points. CDPRs have several advantages such as a high payload-to-weight ratio, a potentially very large workspace, and reconfiguration capabilities. Therefore, they can be used in several applications, e.g. heavy payload handling and airplane painting [2], cargo handling [3], warehouse applications [4], and large-scale assembly operations [5]. Other possible applications include the broadcasting of sport events, haptic devices $[6,7,8]$, support structure for giant telescopes [9, 10], and search and rescue deployable platforms $[11,12]$.

In the sequel, a CDPR configuration refers to the positions of the cable exit points, the positions of the cable anchor points on the moving platform, and the cable layout between these two sets of points. In most previous studies, the CDPR has a fixed configuration which is determined during its design, e.g. [13]. While fixed-configuration CDPRs are relevant in many cases, some more demanding applications require reconfiguration capabilities. One notable case is a cluttered environment where cable collisions with objects in the CDPR workspace cannot be completely avoided so that reconfigurations are necessary. This case is dealt with in this paper where the considered applications involve low force operations over the surface of a large (metallic) structure, e.g. painting and sandblasting. The CDPR environment is cluttered because the structure is located into the workspace and occupies a significant part of it, as illustrated in Fig. 1. The task to be performed is simplified to that of following a prescribed path which is defined by the user in such a way that the entire surface of the structure is eventually treated. The platform orientation is constant. Tools and/or active devices have to be embarked on the CDPR moving platform but the corresponding issues are out of the scope of this paper.

Because of cable collisions, several CDPRs would need to be installed around the structure, typically one per structure face [1]. Alternatively, to reduce the number of winches and thus the installation and maintenance costs, a limited number of winches can be used when reconfigurations are allowed. The most efficient way of avoiding cable collisions is to permit CDPR cable exit point reconfigurations. Exit point reconfigurations can be performed in a continuous or in a discrete manner. The first one consists in cable exit points mounted on mobile bases, e.g. a trolley on a rail [14] or a flying platform [15, 16, 17]. Such a Reconfigurable Cable-Driven Parallel Robot (RCDPR) has a continuous set of possible configurations. On the contrary, the set of possible reconfigurations is discrete when the cable exit points can be positioned at a possibly large but finite number of locations, such as those of a grid of possible

\footnotetext{
${ }^{1}$ Evaluation des CApacités de la RObotique à CÂbles dans un contexte industriel
} 
exit point positions. From a technical point of view, modifying a cable exit point amounts to move or change the last pulley which directs the cable toward the moving platform, as shown in Fig. 1. A possible reconfiguration procedure is detailed in [18].

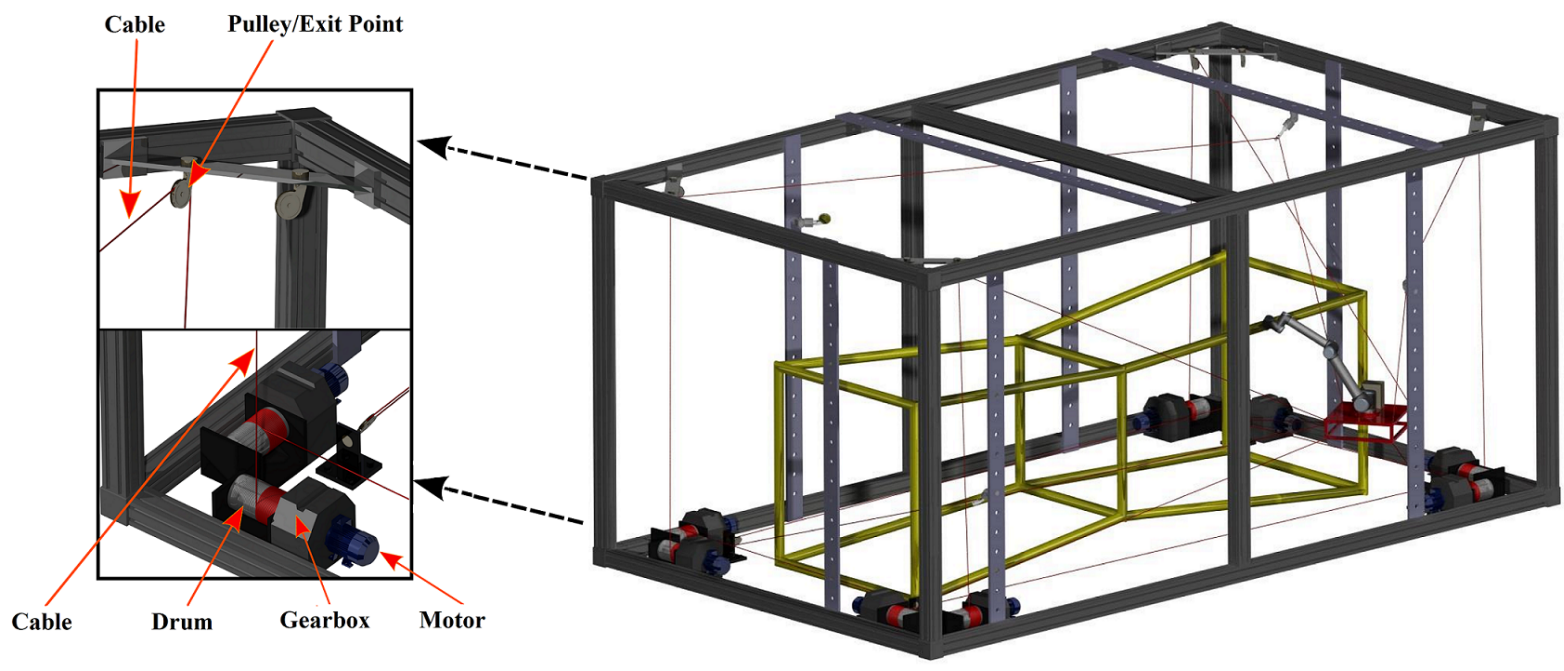

Figure 1: Example of a Reconfigurable Cable-Driven Parallel Robot (RCDPR) design. The robot shown in the picture is a concept created in the framework of the CAROCA project and is intended to paint tubular structures.

Preliminary studies on RCDPRs have already been performed by the NIST as a part of the NIST RoboCrane project [19]. Izard et al. [20] also studied a family of RCDPRs, named ReelAx, in order to investigate the potentialities of CDPRs in industrial contexts. However, no reconfiguration strategy has been proposed by the authors. More detailed studies have been performed by Rosati et al. [21, 22], which focused on planar RCDPRs. They suggested to use movable exit points in order to maximize a local performance index across the CDPR workspace. The corresponding continuous reconfiguration problem is solved by means of an analytical description of the desired optimal cable configuration which can however hardly be extended to spatial RCDPRs. In 2012, Zhou et al. [23] suggested to increase the number of Degrees of Freedom (DOFs) of CDPRs by mounting the winches on mobile bases, which results in a type of RCDPRs. In [24, 25], Zhou used an alternative concept for RCDPRs. A set of cables with constant lengths are connected to linear motors. The cables are either attached to the platform [25] or pull the platform by means of idler pulleys [24]. In both cases, a planar case study has been investigated to show the concept advantages.

Recently, Nguyen et al. [14] proposed a reconfiguration strategy which consists in solving two sub-optimization problems. The first problem aims at defining bounds on the reconfiguration parameter such that the CDPR reconfiguration is formulated as a box-constrained optimization problem. The latter forms the second sub-optimization problem which, for example, can be solved by means of gradient based optimization algorithms.

In these previous works, which all deal with continuous CDPR reconfigurations, two general problems are tackled. The first problem is related to CDPR design optimization. It consists in determining reconfiguration parameter values 
allowing the RCDPR to work adequately across a prescribed workspace. The RCDPR is then only occasionally reconfigured. The second problem is to continuously resolve the reconfiguration parameter values so as to optimize some performance criterion along a prescribed trajectory. In the present paper, discrete reconfigurations are considered because the use of continuous reconfigurations for industrial applications over large structures can be prohibitive since several additional motorized DOFs must be used in order to continuously reconfigure the cable exit points. Hence, the aforementioned second problem is not dealt with in this paper since the set of possible cable exit point positions considered here is discrete and not continuous. The first problem is relevant to a discrete reconfiguration problem. However, because of the cluttered environment considered in the present paper, one configuration can hardly allow the RCDPR mobile platform to follow the whole prescribed path without cable collisions so that several reconfigurations are generally required. To the best of our knowledge, in the case of a discrete set of possible configurations, the issue of optimizing the sequence of reconfigurations which allow the RCDPR mobile platform to follow a prescribed path has never been dealt with before.

Therefore, in the context of a cluttered environment which precludes the use of a fixed-configuration CDPR, this paper introduces means to select and optimize the sequence of discrete reconfigurations permitting the mobile platform of a RCDPR to follow a prescribed path. Quasi-static working conditions are considered. The prescribed path is discretized into a finite set of points. The cable exit points of the RCDPRs under study can be positioned at the points of a user-defined grid of possible locations. The user also defines a set of constraint functions which ensure a proper functioning of the RCDPR. The prescribed path is deemed to be successfully followed if all these constraints are satisfied at all points of the discretized path. As typical examples, this paper considers cable collision, wrench-feasibility and pose error constraint functions.

The paper contribution can then be summarized as follows. A so-called feasibility map is firstly generated. For each possible RCDPR configuration, this map stores the feasibility of the points of the prescribed path. A point is feasible if the corresponding platform pose satisfies all constraint functions, it is unfeasible otherwise. From this map, feasibility transitions are then defined. These transitions represent the reconfigurations that can be possibly performed along the prescribed path. The feasibility map is next analyzed in order to find so-called dominant configurations and then to determine minimum sets of configurations which allow the RCDPR platform to follow the whole prescribed path while satisfying all constraint functions, resulting in a reduced feasibility map. This analysis aims at reducing the number of configurations to be considered in the next step. This next step uses the feasibility transitions to build a graph whose nodes represent the feasible RCDPR reconfigurations along the prescribed path and whose arcs are weighted by means of a user-defined cost function. Some possible cost functions are presented, a relevant example being the number of cable exit point changes required for a given reconfiguration. Finally, the graph is searched using Dijkstra's algorithm which eventually determines the optimal reconfiguration strategy, i.e., the sequence of feasible reconfigurations that permit to follow the whole prescribed path while optimizing a cost function.

Preliminary results of this work have been presented by the authors in the conference publication [18]. Compared to it, the present paper contains a spatial case study (Section 4), while the conference presentation only contained a 
planar one. It also provides more detailed discussions of the proposed discrete reconfiguration strategy (Section 3). Moreover, the feasibility map analysis aiming at reducing the number of configurations used in building the graph is new (Section 3.7).

The paper is organized as follows. Section 2 describes the CDPR kinematics and elasto-static models used in this work. Section 3 describes the main steps of the proposed discrete reconfiguration planning method, detailing the feasibility map computation, its analysis to reduce the number of considered configurations, the graph building, and the graph search for an optimal discrete reconfiguration strategy. Section 4 presents a spatial case study corresponding to the painting and/or sandblasting of a tubular structure. Some conclusions and future works are finally given in Section 5 .

\section{Kinematics and Elasto-Static Model}

\subsection{Notations and Inverse Kinematics}

Figure 2 depicts a RCDPR. The moving platform is actuated by $m$ cables. The $i$-th cable connection point on the platform is denoted by $B_{i}$. The cables are actuated by winches, fixed on the base of the robot. The cables are routed to exit points by means of pulleys. The $i$-th exit point, for a given configuration $C$, is defined as $A_{i, c} . \mathscr{F}_{b}$, of origin $O_{b}$ and axes $\mathbf{x}_{b}, \mathbf{y}_{b}, \mathbf{z}_{b}$ denotes a fixed reference frame. Vector $\mathbf{b}_{i}^{p}$ denotes the position vector of point $B_{i}$ expressed in the moving platform frame $\mathscr{F}_{p}$ of origin $O_{p}$ and axes $\mathbf{x}_{p}, \mathbf{y}_{p}$ and $\mathbf{z}_{p}$. Vector $\mathbf{a}_{i, c}^{b}$ denotes the position vector of point $A_{i, c}$ expressed in the base frame $\mathcal{F}_{b}$.

The Inverse Kinematics (IK) of the RCDPR provides the lengths $l_{i, c} i=1, \ldots, m$ of the cables, for a given configuration of the CDPR and a given pose $\mathbf{p}=[\mathbf{t}, \boldsymbol{\phi}]^{\mathrm{T}}$ of its platform. $\mathbf{t}$ is the position vector of the platform center of mass (CoM) in $\mathscr{F}_{b}$ and $\phi$ contains angles, $\alpha, \beta$ and $\gamma$, which define the platform orientation with respect to $\mathscr{F}_{b}$. Here, the cable weight is neglected and the cables are thus considered to be straight line segments. The length of the $i$-th cable is then given by $l_{i, c}=\left\|\mathbf{a}_{i, c}^{b}-\mathbf{t}-\mathbf{R} \mathbf{b}_{i}^{p}\right\|_{2}$ where $\mathbf{R}=\mathbf{R}(\boldsymbol{\phi})$ the rotation matrix defining the platform orientation.

\subsection{Static Model}

During the task, an external wrench $\mathbf{w}_{e}$ is exerted on the platform. This wrench includes the weight of the platform, the weight of the embarked tools and the force and moments produced by the manufacturing process. The external wrench $\mathbf{w}_{e}$ is a 6-dimensional vector expressed in frame $\mathcal{F}_{b}$ and takes the form $\mathbf{w}_{e}=\left[\mathbf{f}_{e}^{\mathrm{T}}, \mathbf{m}_{e}^{\mathrm{T}}\right]^{\mathrm{T}}=$ $\left[f_{x}, f_{y}, f_{z}, m_{x}, m_{y}, m_{z}\right]^{\mathrm{T}}$ where $f_{x}, f_{y}$ and $f_{z}$ are the $x, y$ and $z$ components of the external force vector $\mathbf{f}_{e} . m_{x}, m_{y}$ and $m_{z}$ are the $x, y$ and $z$ components of the external moment vector $\mathbf{m}_{e}$. The components of the external wrench $\mathbf{w}_{e}$ are assumed to be bounded as follows:

$$
\begin{gathered}
\underline{f} \leq f_{x}, f_{y}, f_{z} \leq \bar{f} \\
\underline{m} \leq m_{x}, m_{y}, m_{z} \leq \bar{m}
\end{gathered}
$$




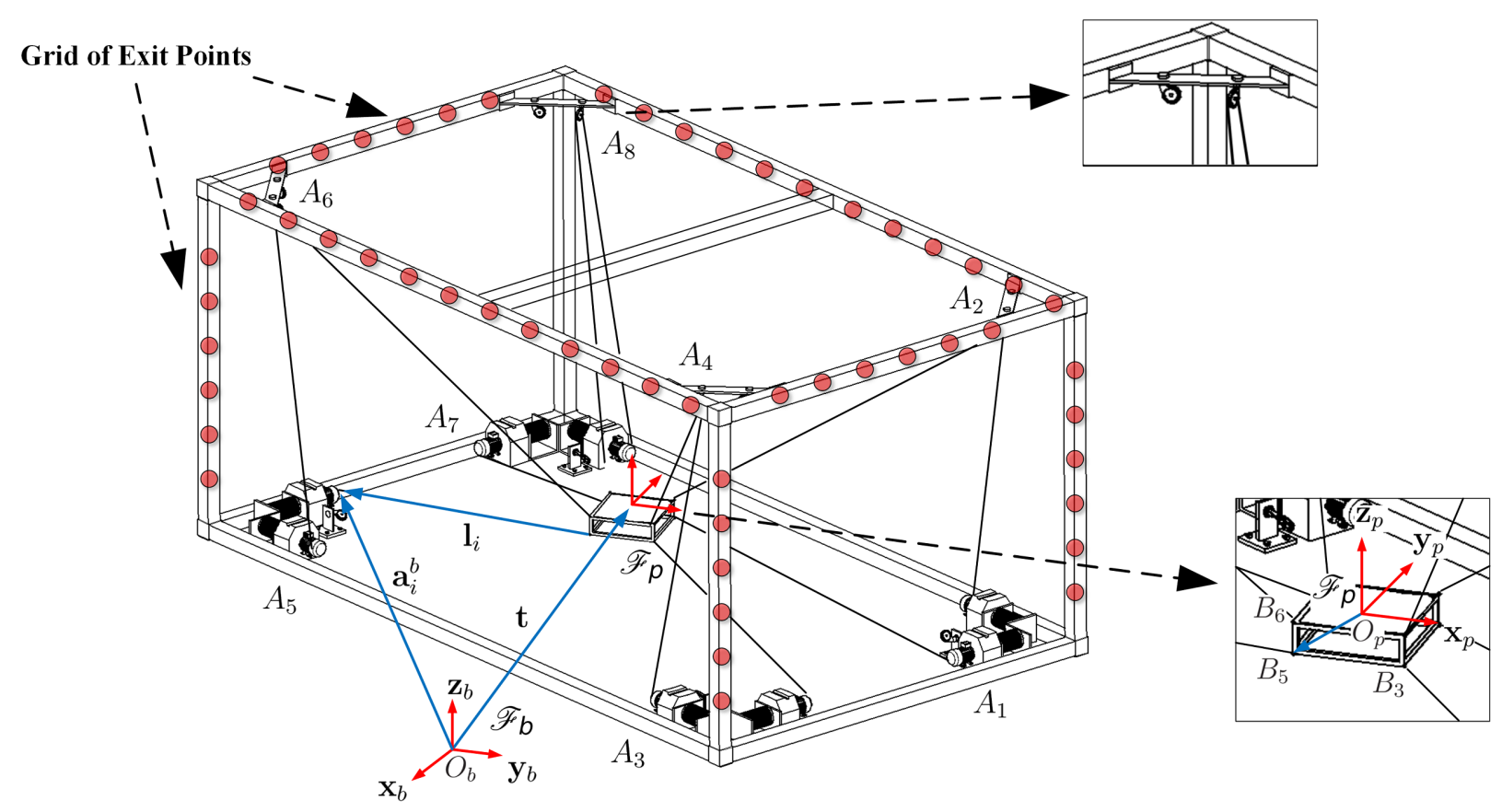

Figure 2: Schematic of a RCDPR. The red points represent the possible locations of the cable exit points, where the pulleys can be fixed.

In order to balance the external wrench, each cable generates on the platform a wrench, proportional to its tension $\tau_{i}=1, \ldots, m$. Hereafter, according to the required operations and assuming that the dynamic forces transmitted to the platform by the embarked tools are negligible, the motion of the CDPR platform is considered quasi-static. The cables balance the external wrench $\mathbf{w}_{e}$, according to the following equation [26]:

$$
\mathbf{W} \boldsymbol{\tau}+\mathbf{w}_{e}=0
$$

where $\tau=\left[\tau_{1}, \ldots, \tau_{m}\right]$ is the vector whose components are the cable tensions $\tau_{i}$ and $\mathbf{W}$ is the wrench matrix.

The RCDPRs considered in this paper are redundantly actuated, i.e., the number of cables is greater than the number of DOFs of the moving platform. Then, the direct static problem (determination of $\tau$ in (3)) generally leads to an infinite number of solutions. In this paper, we used the solution which minimizes the 2-norm of the cable tension vector $\tau$.

\subsection{Elasto-Static Model}

In order to quantify the stiffness of the RCDPR, an elasto-static model is used:

$$
\delta \mathbf{w}_{e}=\mathbf{K} \delta \mathbf{p}
$$

where the matrix $\mathbf{K}$, whose expression is given in [27], defines the relation between an infinitesimal change in the external wrench, $\delta \mathbf{w}_{e}$, and the corresponding infinitesimal change in the pose of the RCDPR platform, $\delta \mathbf{p}=\left[\delta \mathbf{t}^{\mathrm{T}}, \delta \boldsymbol{\phi}^{\mathrm{T}}\right]^{\mathrm{T}}$. 


\section{Reconfiguration Planning Method}

The user provides a grid of possible exit point locations where the cables can be attached to the base frame. The task to be performed can be realized by combining several configurations, disconnecting one or more cable(s) from their exit points and connecting them to new locations. Accordingly, in order to determine the optimal reconfiguration scheme of a RCDPR based on some user defined criteria, a 10-step algorithm, shown in Fig. 3, is explained in the following sub-sections.

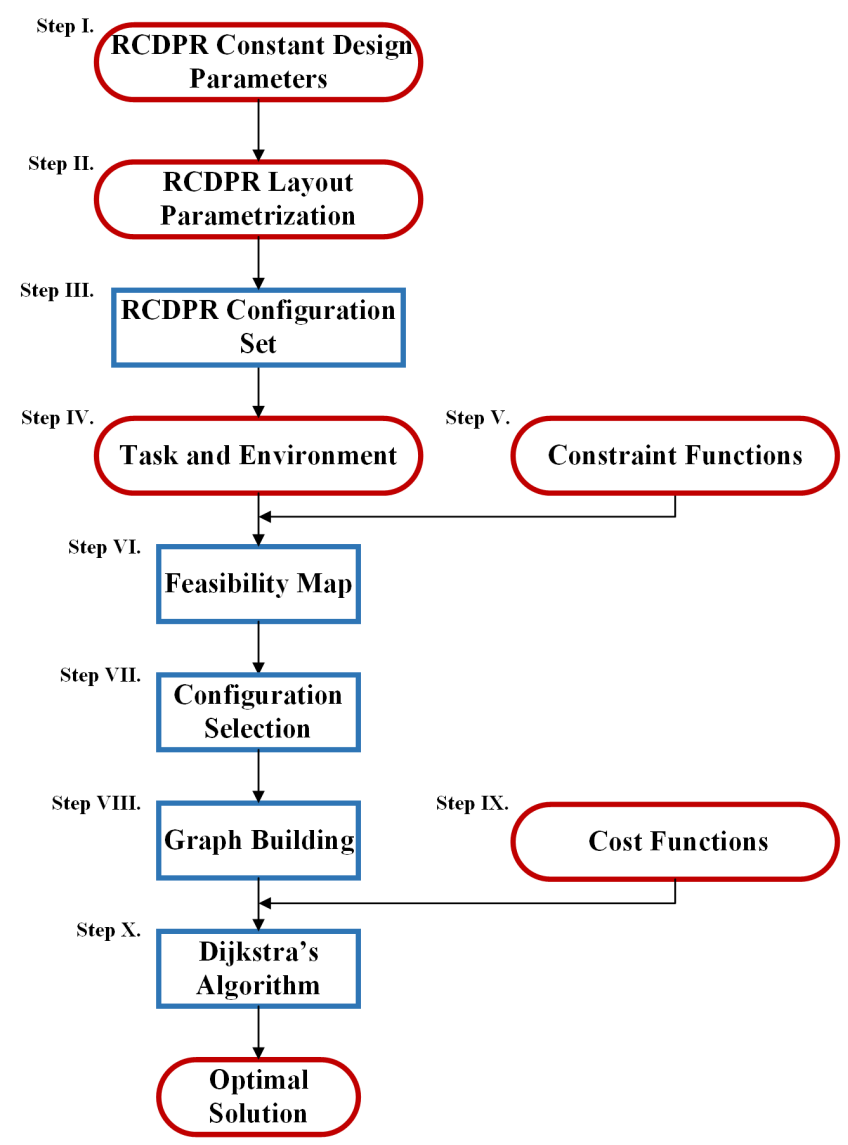

Figure 3: Scheme of the optimal reconfiguration planning method.

\subsection{Step I: Constant Design Parameters}

The user defines the constant design parameters of the RCDPR model, including:

- The number of cables, $m$.

- The cable properties, namely, the Young Modulus, $E$, the diameter, $\phi_{c}$, and the linear stiffness coefficient, $k$.

- The data of the motorization, including the nominal torque, $\tau_{M}$, the nominal speed, $\omega_{M}$, the gearbox transmission ratio, $\rho_{R}$, and the winch diameter, $\phi_{W}$. 
- The positions of the cable connection points on the platform, $B_{i}$, expressed in the local reference frame $\mathscr{F}_{p}$ by the Cartesian coordinate vectors $\mathbf{b}_{i}^{p}$.

\subsection{Step II: RCDPR Layout Parametrization}

The user provides a point grid where the RCDPR exit points can be located. While placing the exit points at the points of this grid, a huge number of CDPR configurations can be generated. In order to limit the complexity of the problem, the user can parametrize the cable layouts, correlating the relative position of the cable exit points, $A_{i, c}, i=1, \ldots, m$, with respect to each other and with respect to the reference frame $\mathscr{F}_{b}$. For instance, the cable exit points can be disposed at the vertices of a parallelepiped, as shown in Fig. 4. The Cartesian coordinates vectors $\mathbf{a}_{i, c}^{b}, i=1, \ldots, m$, are described by means of $n_{v}$ design parameters, $u_{v}, v=1 \ldots, n_{v}$. In the previous example, three design parameters, $x_{1}, x_{2}$, and $x_{3}$ are sufficient to describe the exit point locations.

Once the exit point locations are parametrized, a set of discrete values, $[u]_{v}$, is assigned to each design parameter. The number of values contained in the $v$-th set is equal to $\epsilon_{v}$. The discrete values $[u]_{v}, v=1, \ldots, n_{v}$ are defined according to the grid where the cable exit points can be located.
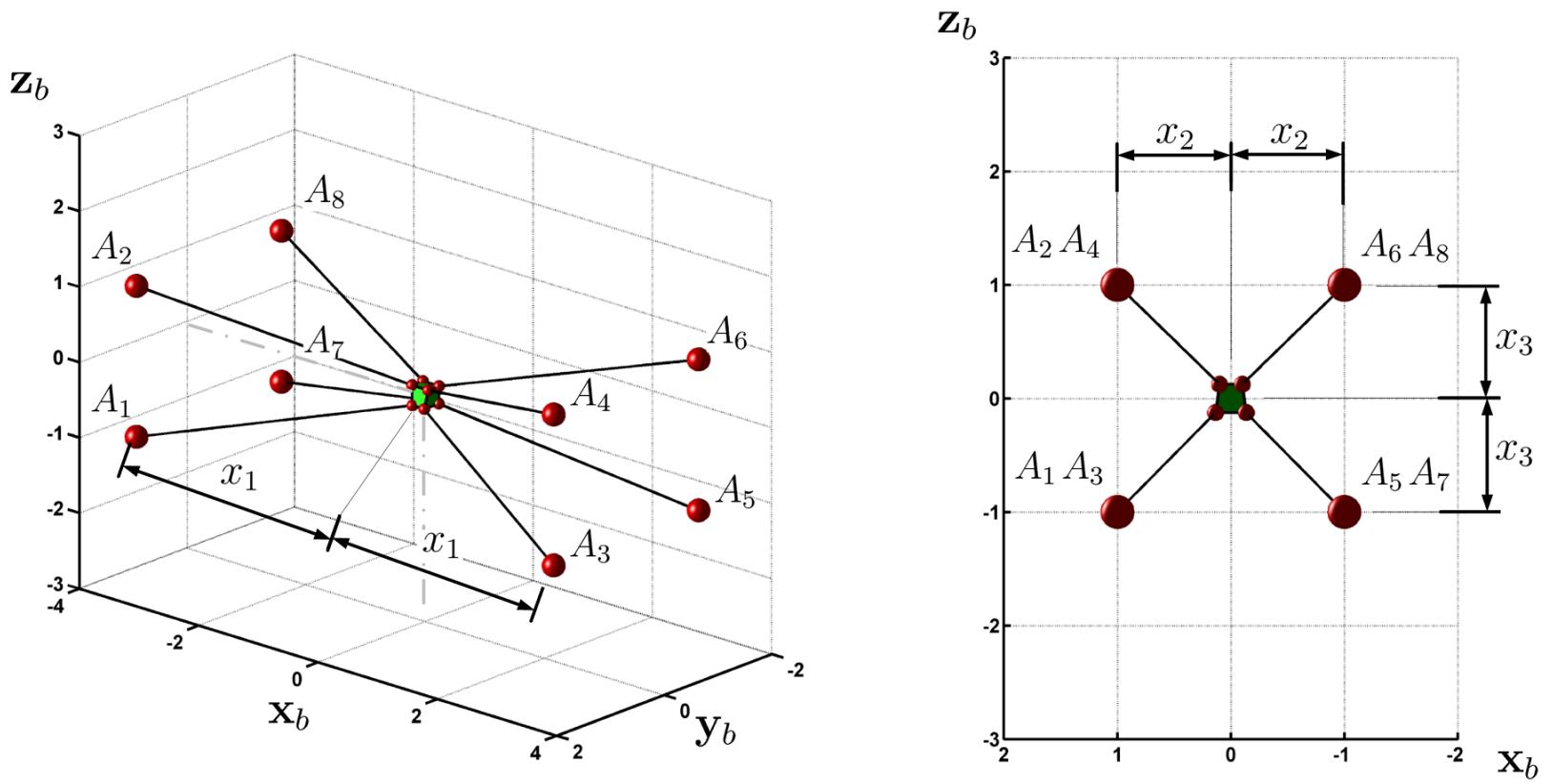

Figure 4: Location and parametrization of the exit points of a RCDPR, disposed on a parallelepiped layout.

\subsection{Step III: RCDPR Configuration Set}

The algorithm combines the discrete values $[u]_{v}$ provided at Step II. Hence, $n_{C}=\prod_{v=1}^{n_{v}} \epsilon_{v}$ configurations of CDPRs are generated. Each $j$-th configuration, $C_{j}$, is described by a design parameter vector, $\mathbf{x}_{j}$, containing the design parameters $u_{v}, v=1 \ldots, n_{v}$ associated to this specific configuration. The configurations are analyzed in the subsequent steps of the algorithm. 


\subsection{Step IV: Task and Environment}

The user provides the data related to the task to be performed and the robot environment, namely:

- The platform weight $\mathbf{w}_{g}$ and the bounds on the external wrench applied on the platform, $\underline{f}, \bar{f}, \underline{m}, \bar{m}$.

- The working environment, modeled by means of simple geometric shapes or approximated by means of polygonal meshes.

- A set of $n_{v p}$ via points. The via points are connected with each other by segments. All the segments should be followed by the RCDPR moving platform CoM. The minimization of the length of the corresponding path is a routing inspection problem [28]. The via points and the segments connecting them are represented as a graph. The graph is modified in such a way to contain at least one Eulerian path (a trail which crosses every edge exactly once) or an Eulerian cycle (an Eulerian path starting and ending at the same vertex). The graph is solved by means of Fleury's algorithm [28]. The user can select one of the solutions, representing the path that the platform of the RCDPR must follow during the task. The chosen path is discretized into $n_{P}$ points, $\mathscr{P}=\left\{P_{i}, i=1, \ldots, n_{P}\right\}$, where $P_{i}, i=1, \ldots, n_{P}$, are the moving platform poses associated to these points. The platform orientation associated to each pose is defined by the user. Figure 5 presents an example of such a path generation in a case where the moving platform has to follow 8 segments starting from the via point $N_{1}$.
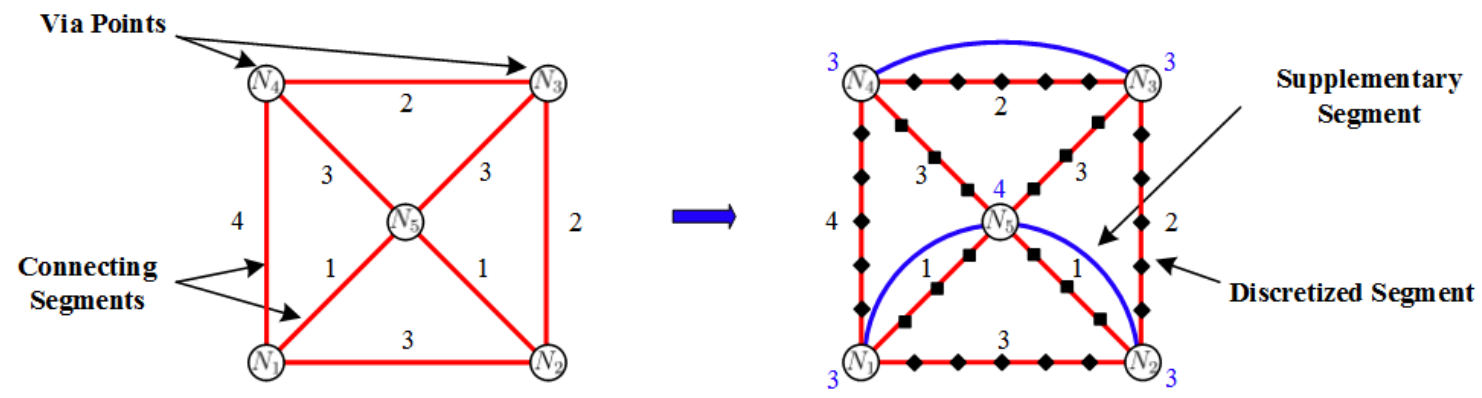

Figure 5: Computation of one of the possible optimal paths covering the whole set of prescribed segments. In this example, the segments to be followed do not represent an Eulerian graph. To transform it into an Eulerian graph, minimizing the cost of the corresponding Eulerian cycles, it is necessary to add three segments (supplementary segments). An optimal path consists in visiting the via points in the following order: $N_{1} N_{5} N_{2} N_{3} N_{5} N_{4} N_{3} N_{4} N_{1} N_{5} N_{2} N_{1}$

\subsection{Step V: Constraint Functions}

The user specifies a set of $n_{\phi}$ constraint functions that the RCDPR should satisfy during the task. Some constraints are presented hereafter.

\subsubsection{Wrench Feasibility}

Since cables can only pull on the platform, the tensions in the cables must always be non-negative. Moreover, cable tensions must be lower than an upper bound, $\tau_{\max }$, which corresponds either to the maximum tension $\tau_{\max 1}$ the cables (or other mechanical parts) can bare, or to the maximum tension $\tau_{\max 2}$ the motors can provide. 
The cable tension bounds can thus be written as:

$$
0 \leq \tau_{i} \leq \tau_{\max }, \quad \forall i=1, \ldots, 8
$$

where $\tau_{\max }=\min \left\{\tau_{\max 1}, \tau_{\max 2}\right\}$.

Due to the cable tension bounds, RCDPRs can balance only a bounded set of external wrenches. This set of wrenches is a convex polytope defined hereafter as the available wrench set, $\left[\mathbf{w}_{e}\right]_{a}$. In order to remain in static equilibrium, the robot should be configured in such a way that the prescribed path is included into the Wrench Feasible Workspace (WFW). The WFW is the set of the wrench feasible poses of the RCDPR. A pose is wrench feasible when the robot is able to balance any external wrench $\mathbf{w}_{e}$ whose components satisfy (1) and (2) [29]. These wrenches compose the so-called required wrench set, which is here an hyper-rectangle $\left[\mathbf{w}_{e}\right]_{r}$. Wrench feasibility amounts to the inclusion of the hyper-rectangle $\left[\mathbf{w}_{e}\right]_{r}$ into the polytope $\left[\mathbf{w}_{e}\right]_{a}$. This condition can be expressed by the following set of inequalities:

$$
\mathbf{C w}_{e} \leq \mathbf{d}, \quad \forall \mathbf{w}_{e} \in\left[\mathbf{w}_{e}\right]_{r}
$$

where matrix $\mathbf{C}$ and vector $\mathbf{d}$ can be computed as detailed in [29] and [30].

\subsubsection{Cable Interferences}

A second constraint is related to the possible collisions between cables. If two or more cables collide, the geometric and static models of the CDPR are not valid anymore and the cables can be damaged or their life time severely reduced.

In order to verify that cables do not interfere, it is sufficient to determine the distances between them. Modeling the cables as linear segments, the distance $d_{i, j}^{c c}$ between the $i$-th cable and the $j$-th cable can be computed, e.g. by means of the method presented in [31]. There is no interference if the distance is larger than the diameter of the cables, $\phi_{c}$ :

$$
d_{i, j}^{c c} \geq \phi_{c} \quad \forall i, j=1, \ldots, m, \quad i \neq j
$$

The number of possible cable interferences to be verified is equal to $C_{2}^{m}=\frac{m !}{2 !(m-2) !}$. Note that, depending on the way the cables are routed from the winches to the moving platform, possible interferences of the cable segments between the winches and the pulleys may have to be considered.

\subsubsection{Collisions between the Cables and the Environment}

Industrial environments may be cluttered. Collisions between the environment and cables of the RCDPR should be avoided. For fast collision detection, the environment objects are modeled with basic shapes such as spheres and cylinders. When more complex shapes have to be considered, their surfaces are approximated with polygonal meshes. Thus, the collision analysis is performed by computing the distances between the edges of those polygons and the cables, e.g. by using [31]. 


\subsubsection{Pose Error}

Cables are not perfectly rigid body. Consequently, the cables are subjected to some deformations that may induce some moving platform pose errors. $\delta \mathbf{t}=\left[\delta t_{x}, \delta t_{y}, \delta t_{z}\right]^{\mathrm{T}}$ and $\delta \boldsymbol{\phi}=[\delta \gamma, \delta \beta, \delta \alpha]^{\mathrm{T}}$ characterize the positioning and orientation errors of the moving platform, respectively (Section 2.3).

The pose error must remain bounded according to a positioning error threshold vector, $\delta \mathbf{t}_{c}=\left[\delta t_{x, c}, \delta t_{y, c}, \delta t_{z, c}\right]$, where $\delta t_{x, c}, \delta t_{y, c}$ and $\delta t_{z, c}$ are bounds on the positioning errors along the axes $\mathbf{x}_{b}, \mathbf{y}_{b}$ and $\mathbf{x}_{b}$, and an orientation error threshold vector, $\delta \boldsymbol{\phi}=\left[\delta \gamma_{c}, \delta \beta_{c}, \delta \alpha_{c}\right]$, where $\delta \gamma_{c}, \delta \beta_{c}$ and $\delta \alpha_{c}$ are bounds on the platform orientation errors about the axes $\mathbf{x}_{b}, \mathbf{y}_{b}$ and $\mathbf{z}_{b}$, i.e.:

$$
\begin{gathered}
-\left[\delta t_{x, c}, \delta t_{y, c}, \delta t_{z, c}\right] \leq\left[\delta t_{x}, \delta t_{y}, \delta t_{z}\right] \leq\left[\delta t_{x, c}, \delta t_{y, c}, \delta t_{z, c}\right] \\
-\left[\delta \gamma_{c}, \delta \beta_{c}, \delta \alpha_{c}\right] \leq[\delta \gamma, \delta \beta, \delta \alpha] \leq\left[\delta \gamma_{c}, \delta \beta_{c}, \delta \alpha_{c}\right]
\end{gathered}
$$

\subsection{Step VI: Feasibility Map}

This step aims at identifying the RCDPR configurations $C_{j}, j=1, \ldots, n_{C}$ which, at a given point $P_{i} \in \mathscr{P}$, satisfies all the constraints defined at Step V. In particular, any pose associated to the $i$-th point, $P_{i}$, and the $j$-th configuration, $C_{j}$, is said to be feasible if it satisfies all the $n_{\phi}$ user-defined constraints, $\phi_{l}(i, j), l=1, \ldots, n_{\phi}$. The information associated to the pose feasibility is stored in the variables $F_{i, j}, i=1, \ldots, n_{p}, j=1, \ldots, n_{C}$ where $i$ and $j$ represent the index of the analyzed point and the index of the analyzed configuration, respectively.

\section{Definition 1.}

$$
F_{i, j}=1 \quad \Longleftrightarrow \quad \sum_{l=1}^{n_{\phi}} \phi_{l}(i, j)=0
$$

where $\phi_{l}(i, j), l=1, \ldots, n_{\phi}$ is defined as :

$$
\left\{\begin{array}{l}
\phi_{l}(i, j)=0 \quad \Longleftrightarrow \quad \text { the } l \text {-th constraint is satisfied for the } j \text {-th robot configuration at point } P_{i} \\
\phi_{l}(i, j)=1 \quad \Longleftrightarrow \quad \text { the } l \text {-th constraint is not satisfied for the } j \text {-th robot configuration at point } P_{i}
\end{array}\right.
$$

A feasibility map is created according to the values of $F_{i, j}, i=1, \ldots, n_{P}, j=1, \ldots, n_{C}$. A simple feasibility map is shown in Fig. 6. The horizontal axis represents the points $P_{i}, i=1, \ldots, n_{P}$ of the discretized prescribed path. The vertical axis shows the values of $F_{i, j}$ computed for four configurations $C_{1}, C_{2}, C_{3}$ and $C_{4}$. At a given point $P_{i}, F_{i, j}=1$ if the pose assumed by configuration $C_{j}$ is feasible and $F_{i, j}=0$ if the pose assumed by configuration $C_{j}$ is infeasible. From the feasibility map, it is apparent that, for a given configuration, several changes in the feasibility conditions are possible between two consecutive points $P_{i}$ and $P_{i+1}$. These feasibility changes are named Feasibility Transitions (FT). Hereafter, $F_{i, j}$ is said to be a Positive Feasibility Transition (PFT) when a transition from an infeasible condition to a feasible one occurs at point $P_{i}$ for configuration $C_{j}$. 


\section{Definition 2.}

$$
F_{i, j} \text { is a PFT if }\left\{\begin{array}{l}
F_{i, j}=1 \\
F_{i-1, j}=0 \\
F_{i+1, j}=1
\end{array}\right.
$$

On the contrary, $F_{i, j}$ is said to be a Negative Feasibility Transition (NFT) when a transition from a feasible condition to an infeasible one occurs.

\section{Definition 3.}

$$
F_{i, j} \text { is a NFT if }\left\{\begin{array}{l}
F_{i, j}=1 \\
F_{i+1, j}=0 \\
F_{i-1, j}=1
\end{array}\right.
$$

For instance, observing the example of Fig. $6, F_{6,3}$ is a PFT since $F_{5,3}=0$ and $F_{6,3}=F_{7,3}=1$. Moreover, $F_{2,3}$ is a NFT since $F_{1,3}=F_{2,3}=1$ and $F_{3,3}=0$. In the following sections, the possible reconfigurations of the RCDPR will be generated only in presence of a PFT or a NFT. In Fig. 6, a so-called isolated feasible transition can be seen at $F_{9,1}$ since the surrounding poses are not feasible, i.e., $F_{8,1}=0$ and $F_{10,1}=0$. Obviously, isolated feasible transitions need not be taken into account during the computation of the optimal reconfiguration strategy.

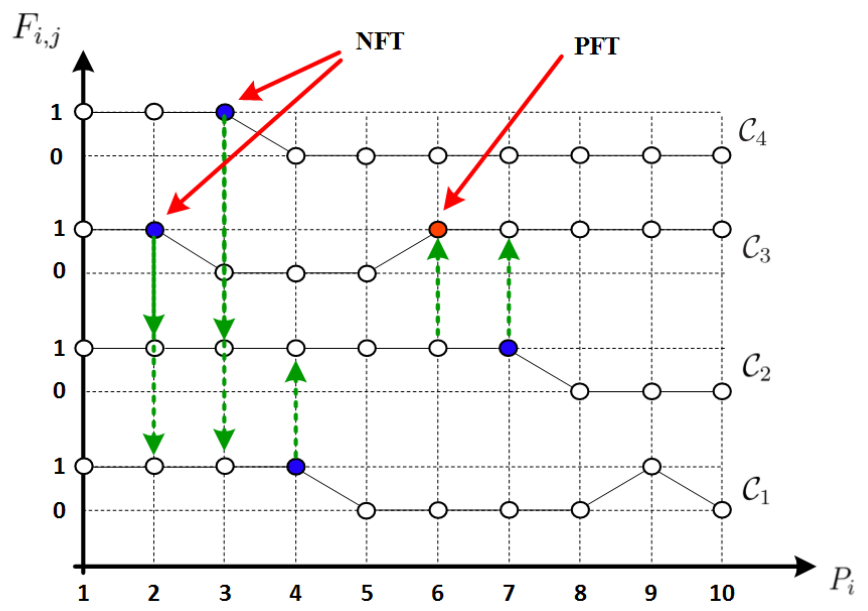

Figure 6: A feasibility map: The green arrows represent the possible reconfigurations, the red dots show the Positive Feasibility Transitions (PFTs), and the blue dots show the Negative Feasibility Transition (NFTs).

\subsection{Step VII: Configuration Selection}

Some feasibility maps may be composed of a large number $n_{C}$ of configurations. The present step aims at reducing the number of configurations of the feasibility map to be used in the following optimization by analyzing the minimum 
number of configurations, $n_{m}$, that can be used to follow the whole prescribed path $\mathscr{P}$. Figure 7 illustrates this configuration selection procedure.

a. Feasibility Map Modification (Fig. 7a). The algorithm simplifies the data stored in the feasibility map. A configuration may present isolated feasible transitions (as illustrated in Sec. 3.6) or isolated clusters of few adjacent feasible poses. In both cases, it is not convenient to use these feasible poses. Isolated feasible poses imply that the RCDPR should be reconfigured to cover only a small portion of the prescribed path of the mobile platform. This situation does not facilitate the achievement of practical goals such as the minimization of the number of reconfigurations to be performed to follow the whole prescribed path. Each isolated feasible transition, $F_{i, j}=1$, s.t. $F_{i-1, j}=0, F_{i+1, j}=0$ is transformed directly into an infeasible pose, $F_{i, j}=0$. Regarding the clusters composed of a few feasible poses, they are detected by analyzing each configuration $C_{j}, j=1, \ldots, n_{\mathcal{C}}$ by means of a clustering algorithm: If the detected cluster contains a number of feasible poses lower than a user defined threshold, $h_{1}$, the poses belonging to this cluster are transformed into infeasible ones.

b. Filtering (Fig. 7b). The algorithm computes the percentage of feasible poses, $p_{j}$, associated to each configuration $C_{j}$ :

$$
p_{j}=\frac{\sum_{i=1}^{n_{P}} F_{i, j}}{n_{P}}
$$

The configurations with a number of feasible poses lower than a user defined threshold, $h_{2}$, are eliminated from the feasibility map. As a result, $n_{C^{\prime}}$ configurations remain in the feasibility map after this filtering process.

c. Dominant Solution Computation (Fig. $7 c$ ). The algorithm analyzes the $n_{C^{\prime}}$ configurations at disposal and identifies the so-called dominated and dominant configurations.

\section{Definition 4.}

A dominated configuration is a configuration $C_{j}$ such that its feasible poses are all included in the set of feasible poses of another configuration $C_{k}$ and whose percentage of feasible poses, $p_{j}$ is strictly lower than $p_{k}$

$C_{j}$ is a dominated configuration if $\exists C_{k}, k \neq j \quad$ s.t. $\left\{\begin{array}{l}\forall F_{i, j}, i=1, \ldots, n_{P}: F_{i, j}=1 \quad \Longrightarrow \quad F_{i, k}=1 \\ p_{j}<p_{k}\end{array}\right.$

Consequently, the dominant configurations can be defined as follows.

\section{Corollary 1.}

A dominant configuration is a configuration such that its feasible poses are not all included in the set of feasible poses of another configuration. 


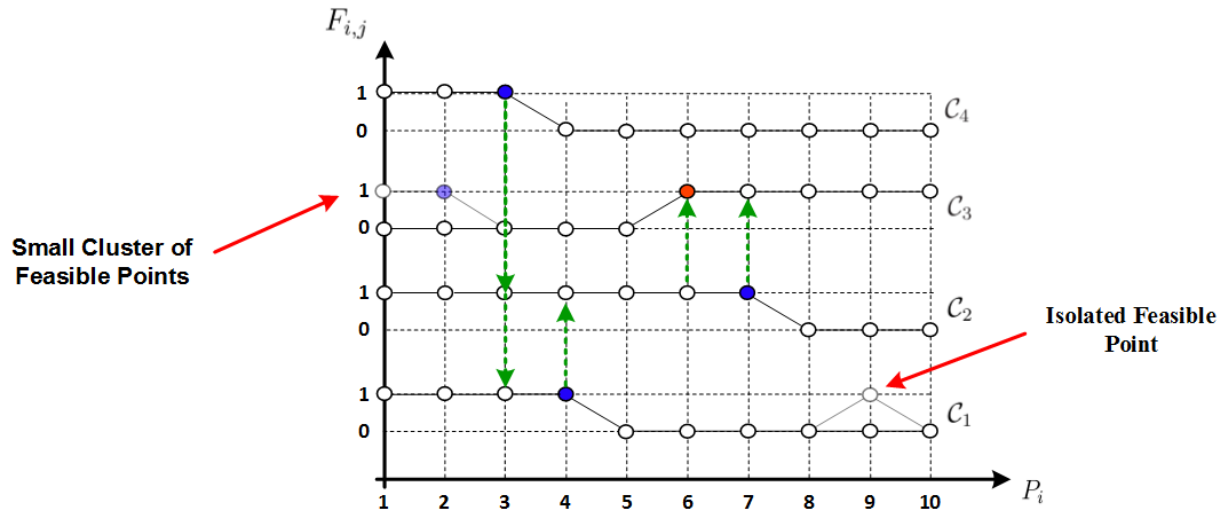

(a) Feasibility map modification.

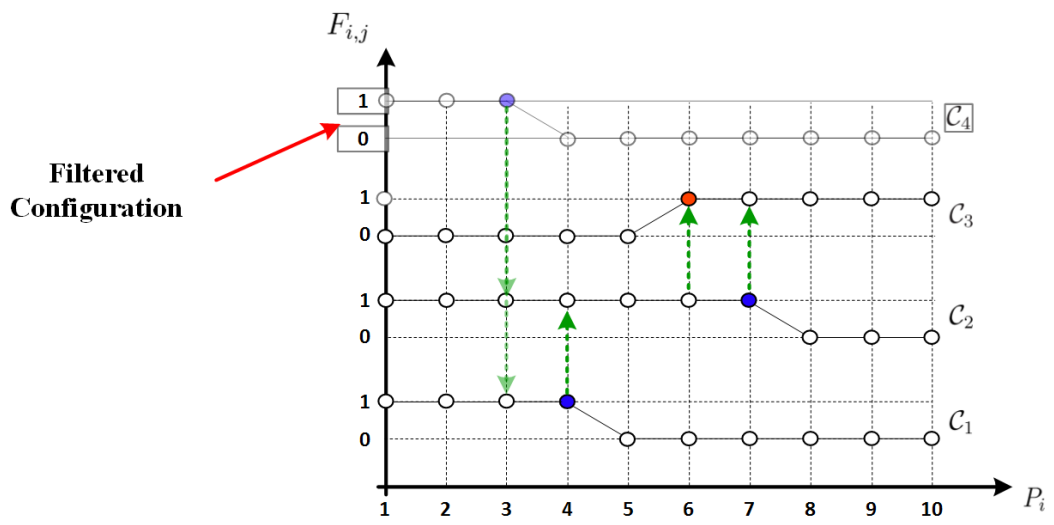

(b) Filtering.

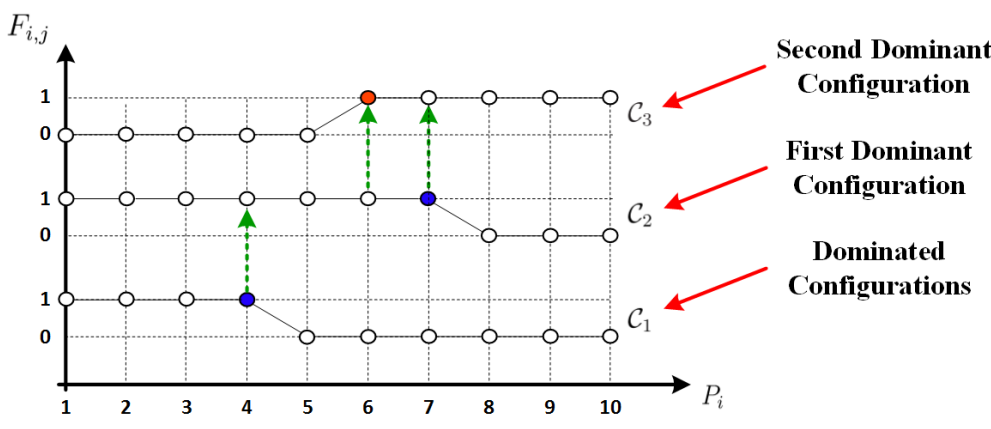

(c) Dominant solution computation.

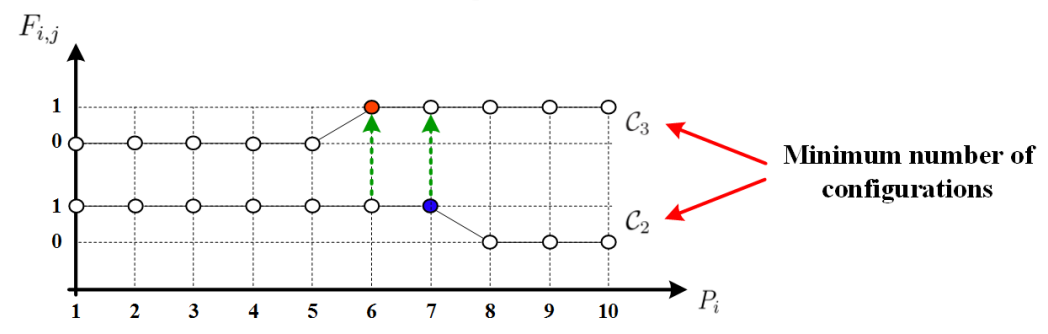

(d) Computation of the minimum number of configurations.

Figure 7: Example of a feasibility map and minimum set of configurations to be used during the graph building. 
d. Computation of the Minimum Number of Configurations (Fig. $7 d$ ). Given $n_{d}$ dominant configurations, the algorithm computes the minimum number of configurations, $n_{m}$, that allow the moving platform to follow the whole prescribed path while satisfying all constraint functions. The computation is performed by analyzing the possible dominant configuration combinations of dimension $\eta$. The analysis starts at $\eta=1$. The value of $\eta$ is increased till at least one combination covers all the points of the prescribed path. For a given $\eta$, the number of combinations to be tested is equal to $C_{\eta}^{n_{d}}=\frac{n_{d} !}{\eta !\left(n_{d}-\eta\right)}$. In the best case, only one configuration is sufficient, $n_{m}=\eta=1$. In the worst case, all dominant configurations are required to follow the complete path, i.e., $n_{m}=\eta=n_{d}$.

e. Definition of the Configurations to Build the Graph. Among all the $C_{n_{m}}^{n_{d}}=\frac{n_{d} !}{n_{m} !\left(n_{d}-n_{m}\right)}$ combinations of $n_{m}$ configurations, all the ones which proved to belong to a combination which covers entirely the prescribed path are retained and will be used to build the graph of the reconfigurations (Step VIII). When the prescribed path cannot be fully covered, due to an extremely cluttered environment or other external constraints, the algorithm selects the set of configurations that let cover the largest number of points of the prescribed path.

An example of the configuration selection procedure, based on a 4-configurations feasibility map, is illustrated in Fig. 7. $C_{1}$ contains an isolated feasibility transition at $P_{9}$ and configuration $C_{3}$ has a cluster of two feasible points at $P_{1}$ and $P_{2}$. With the threshold $h_{1}=3$, the feasibility map is modified in such a way that $F_{9,1}=0, F_{1,3}=0$ and $F_{2,3}=0$ (Fig. 7a). According to the number of feasible points of the four configurations, and with a threshold $h_{2}=0.4$, configuration $C_{4}$ has been eliminated, since $p_{4}=0.3$ (Fig. $7 \mathrm{~b}$ ). The analysis performed over the remaining configurations shows that configuration $C_{1}$ is dominated by configuration $C_{2}$ (Fig. 7c). Configuration $C_{3}$ is also a dominant configuration. Therefore, $C_{2}$ and $C_{3}$ are the two dominant configurations of the problem at hand that can be used to complete the task (Fig. 7d).

\subsection{Step VIII: Graph Building}

A graph is generated from the reduced feasibility map obtained at Step VII. The nodes of the graph, denoted as $N_{i, j, k}$, represent the possible reconfigurations the robot can perform at point $P_{i}$ to switch from the actual feasible configuration $C_{j}$ to another feasible configuration $C_{k}$. The nodes are connected by oriented arcs representing the possible sequences of reconfigurations to be followed in order to follow the whole prescribed path. In fact, two nodes $N_{i, j, k}$ and $N_{i^{\prime}, j^{\prime}, k^{\prime}}$ are adjacent when $i^{\prime}>i, k=j^{\prime}$ and all the poses between $P_{i, k}$ and $P_{i^{\prime}, k}$ are feasible: 


\section{Definition 5.}

$$
N_{i, j, k} \text { and } N_{i^{\prime}, j^{\prime}, k^{\prime}} \text { are adjacent if }\left\{\begin{array}{l}
i^{\prime}>i \\
j^{\prime}=k \\
F_{i^{\prime \prime}, k}=1, \quad \forall i^{\prime \prime} \text { s.t. } i \leq i^{\prime \prime} \leq i^{\prime}
\end{array}\right.
$$

The graph building algorithm is illustrated in Fig. 8. It consists of the following steps.

a. The first node, $N_{0}$, is a virtual node. This node represents the starting point of the graph. It is associated to a virtual point of the prescribed path, $P_{0}$, and it is not assigned to any configuration. Its adjacent nodes, $N_{1,0, k}$, are associated to the point $P_{1}$ and they are created only when a configuration $C_{k}$ is feasible at point $P_{1}$, i.e., $F_{1, k}=1$.

b. The intermediate nodes, $N_{i, j, k}$, are generated by analyzing each feasibility transition of the feasibility map, from point $P_{2}$ to point $P_{n_{p}-1}$. Nodes are generated in correspondence to NFTs and PFTs. Given a NFT, a node $N_{i, j, k}$ is generated if configuration $C_{k}$ is feasible at points $P_{i}$ and $P_{i+1}$.

\section{Definition 6.}

$$
N_{i, j, k} \text { is generated if }\left\{\begin{array}{l}
F_{i, j} \text { is a NFT } \\
F_{i, k}=1 \\
F_{i+1, k}=1
\end{array}\right.
$$

Given a PFT, a node $N_{i, k, j}$ is generated if configuration $C_{k}$ is feasible at points $P_{i}$ and $P_{i-1}$. Thus:

\section{Definition 7.}

$$
N_{i, k, j} \text { is generated if }\left\{\begin{array}{l}
F_{i, j} \text { is a PFT } \\
F_{i, k}=1 \\
F_{i-1, k}=1
\end{array}\right.
$$

Thus, the following corollary is verified:

\section{Corollary 2.}

According to Definitions 6 and 7, a node $N_{i, j, k}$ exists only if both configurations $C_{j}$ and $C_{k}$ are feasible at point $P_{i}: F_{i, j}=1$ and $F_{i, k}=1$ 
c. The ending node, $N_{e}$, is a virtual node. This node is associated to point $P_{n_{p}}$ and it is not assigned to any configuration. Its adjacent nodes, $N_{n_{P}, j, e}$, are associated to point $P_{n_{P}}$ and they are created only when a configuration $C_{j}$ is feasible at point $P_{n_{P}}$, e.g., $F_{n_{P}, j}=1$.

Finally, the adjacent nodes are connected to each other, according to Definition 5, and a cost is assigned to each arc. Some relevant cost functions are discussed in Sec. 3.9. Figure 9 shows the graph associated to the feasibility map given in Fig. 6.

\subsection{Step IX: Cost Functions}

The user defines a set of $n_{\mu}$ cost functions, $\mu_{t}, t=1, \ldots, n_{\mu}$. The cost functions are used to compute the cost of each arc of the graph. The cost functions to be used depend on the task to be performed by the RCDPR or on the user preferences. Nevertheless, five relevant cost functions are presented thereafter.

1. Number of reconfigurations. The first cost function, $\mu_{1}$, bounded between 0 and 1 , aims at minimizing the number of reconfigurations, $n_{r}$, defined as the number of exit point changes to be performed in order to switch from configuration $C_{j}$ to configuration $C_{k}$ :

$$
\mu_{1}=n_{r}
$$

Note that $\mu_{1}$ is equal to $m$ if all the cables have to be reconfigured. This cost function can be considered in order to reduce the number of cable attaching/detaching operations.

2. Capacity margin. The second cost function, $\mu_{2}$, aims at maximizing the capacity margin of the RCDPR [32]. The capacity margin quantifies how "close" the RCDPR is to a non static equilibrium configuration, with respect to the required wrench set, [w] $]_{e}$. Given two adjacent nodes, $N_{i, j, k}$ and $N_{i^{\prime}, k, k^{\prime}}, \mu_{2}$ is defined as the inverse of the average of the capacity margin between points $P_{i}$ and $P_{i^{\prime}}$ of the prescribed path. The evaluation is performed with respect to configuration $C_{k}$ :

$$
\mu_{2}=\frac{\sum_{i^{\prime \prime}=i}^{i^{\prime}} S_{i^{\prime \prime}, k}}{\left(i^{\prime}-i\right)} \frac{\left(i^{\prime}-i\right)}{n_{p}}=\frac{\sum_{i^{\prime \prime}=i}^{i^{\prime}} S_{i^{\prime \prime}, k}}{n_{p}}
$$

where the capacity margin $S_{i^{\prime \prime}, k}$, measured at points $P_{i^{\prime \prime}}, i^{\prime \prime}=i, \ldots, i^{\prime}$ for configuration $C_{k}$, is defined as follows:

$$
S_{i^{\prime \prime}, k}=\min _{j=1, \ldots, n_{j}}\left(\min _{l=1, \ldots, n_{l}}\left(s_{i^{\prime \prime}, j, l}\right)\right)
$$

$s_{i^{\prime \prime}, j, l}$ being the signed distance from the $j$-th vertex of $\left[\mathbf{w}_{e}\right]_{r}$ to the $l$-th face of $\left[\mathbf{w}_{e}\right]_{a} . n_{j}$ and $n_{l}$ are equal to the number of vertices of $\left[\mathbf{w}_{e}\right]_{r}$ and the number of faces of $\left[\mathbf{w}_{e}\right]_{a}$, respectively.

It has to be noted that vector $\mathbf{w}_{e}$ and matrix $\mathbf{W}$ in (3) are not homogeneous. In order to compute the capacity 


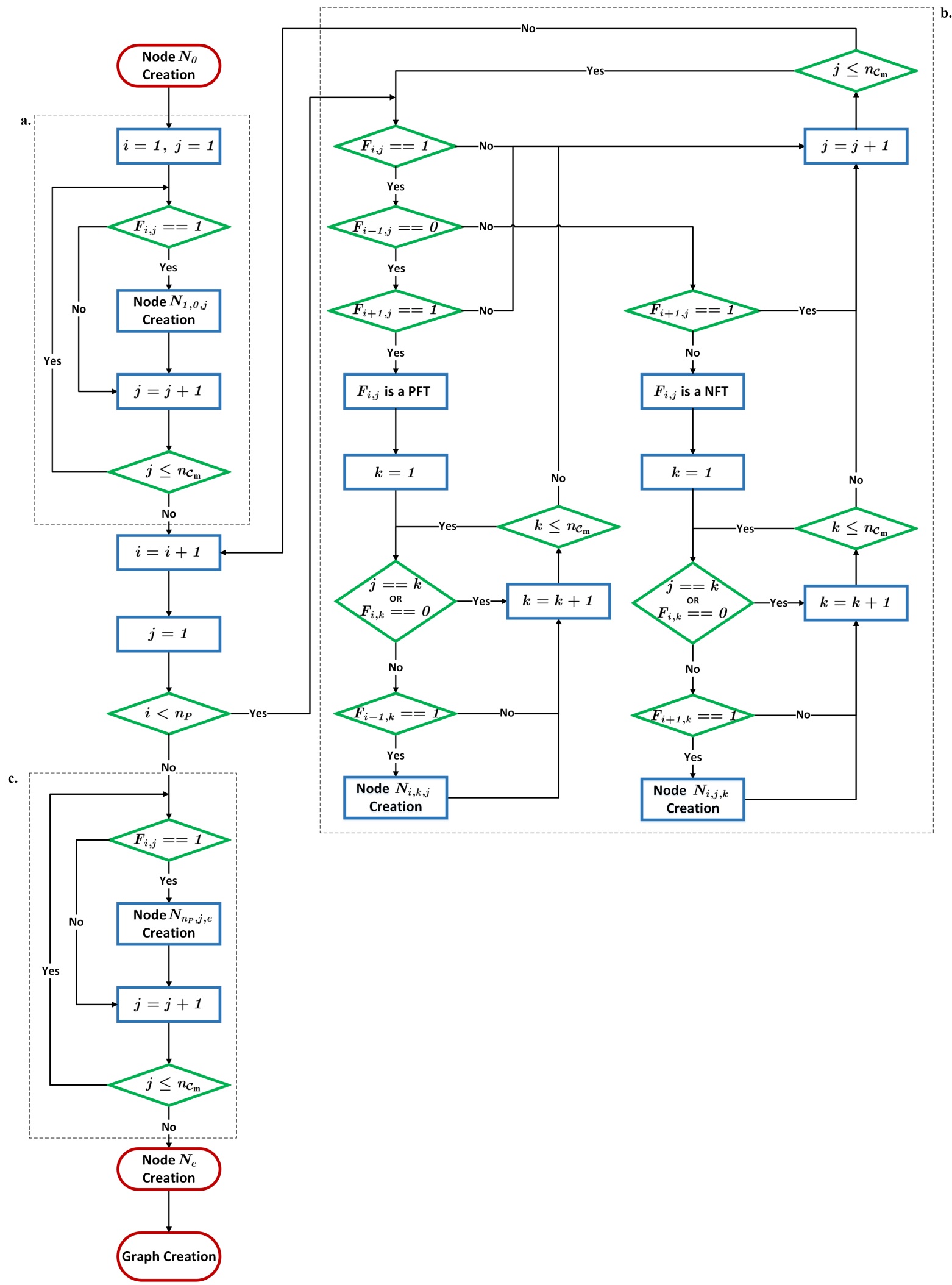

Figure 8: Flowchart of the graph building algorithm. 


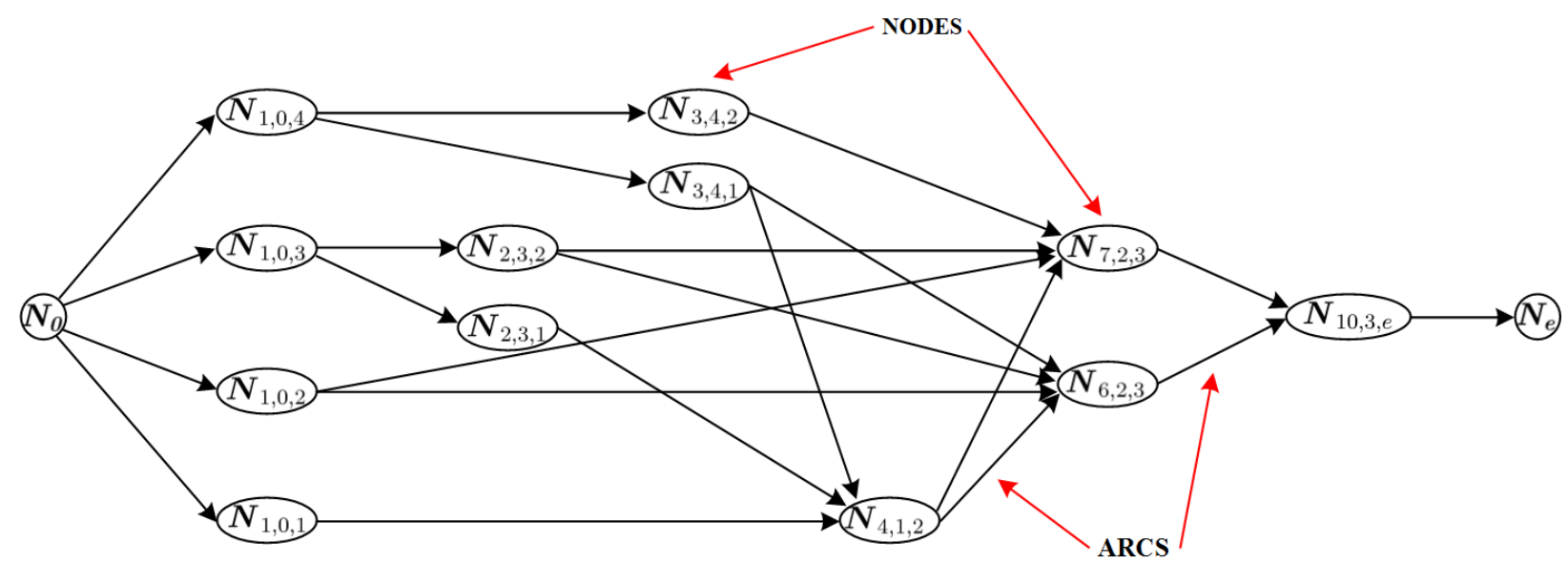

Figure 9: Graph associated to the feasibility map shown in Fig. 6.

margin, they are normalized as follows:

$$
\begin{aligned}
\mathbf{W}_{n} & =\left[\begin{array}{ll}
\mathbb{I}_{3} & \mathbf{0}_{3} \\
\mathbf{0}_{3} & \frac{1}{r_{g}} \mathbb{I}_{3}
\end{array}\right] \mathbf{W} \\
\mathbf{w}_{e, n} & =\left[\begin{array}{ll}
\mathbb{I}_{3} & \mathbf{0}_{3} \\
\mathbf{0}_{3} & \frac{1}{r_{g}} \mathbb{I}_{3}
\end{array}\right] \mathbf{w}_{e}
\end{aligned}
$$

with $r_{g}{ }^{2}=(1 / m) \sum_{i=1}^{m}\left\|\mathbf{b}_{i}^{b}\right\|_{2}^{2}$ is the radius of gyration of the moving-platform. $\mathbb{I}_{3}$ and $\mathbf{0}_{3}$ are the [3x3] identity and null matrices, respectively.

3. Positioning error. The third cost function, $\mu_{3}$, aims at minimizing the norm of the positioning error of the RCDPR moving platform. Given two adjacent nodes, $N_{i, j, k}$ and $N_{i^{\prime}, k, k^{\prime}}$, this cost function is expressed as the weighted mean of the 2-norm of the positioning error, $\overline{\|\delta \mathbf{t}\|_{2}}$, of the moving platform between points $P_{i}$ and $P_{i^{\prime}}$ of the prescribed path. The evaluation is performed with respect to configuration $C_{k}$ :

$$
\mu_{3}=\overline{\|\delta \mathbf{t}\|_{2}} \frac{\left(i^{\prime}-i\right)}{n_{p}}=\frac{\sum_{i^{\prime \prime}=i}^{i^{\prime}}\left\|\delta \mathbf{t}_{i^{\prime \prime}, k}\right\|_{2}}{i^{\prime}-i} \frac{\left(i^{\prime}-i\right)}{n_{p}}=\frac{\sum_{i^{\prime \prime}=i}^{i^{\prime}}\left\|\delta \mathbf{t}_{i^{\prime \prime}, k}\right\|_{2}}{n_{P}}
$$

where $\left\|\delta \mathbf{t}_{i^{\prime \prime}, k}\right\|_{2}$ is the 2-norm of the positioning error vector at point $P_{i^{\prime \prime}}$ for configuration $C_{k}$.

4. Cable tensions. The fourth cost function, $\mu_{4}$, aims at minimizing the cable tensions. Given two adjacent nodes, $N_{i, j, k}$ and $N_{i^{\prime}, k, k^{\prime}}, \mu_{4}$ is equal to the weighted mean of the norm of the cable tension vector, $\overline{\boldsymbol{\tau}} \boldsymbol{\tau}_{2}$, between points $P_{i}$ and $P_{i^{\prime}}$ along the prescribed path. As mentioned in Sec. 2.2, the minimal 2-norm cable tension vector is 
chosen as the solution to (3). The evaluation is performed with respect to configuration $C_{k}$ :

$$
\mu_{4}=\overline{\|\tau\|_{2}} \frac{\left(i^{\prime}-i\right)}{n_{p}}=\frac{\sum_{i^{\prime \prime}=i}^{i^{\prime}}\left\|\tau_{i^{\prime \prime}, k}\right\|_{2}}{i^{\prime}-i} \frac{\left(i^{\prime}-i\right)}{n_{p}}=\frac{\sum_{i^{\prime \prime}=i}^{i^{\prime}}\left\|\tau_{i^{\prime \prime}, k}\right\|_{2}}{n_{p}}
$$

where $\left\|\tau_{i^{\prime \prime}, k}\right\|_{2}$ is the 2-norm of the cable tension vector at point $P_{i^{\prime \prime}}$ for configuration $C_{k}$.

5. Cable tension variations. The fifth cost function, $\mu_{5}$, aims at minimizing the cable tension variations along the prescribed path. This function is defined by the weighted standard deviation $\sigma_{\tau}$ of the norm of the cable tension vector, between points $P_{i}$ and $P_{i^{\prime}}$. The evaluation is performed with respect to configuration $C_{k}$ :

$$
\mu_{5}=\sigma_{\tau} \frac{\left(i^{\prime}-i\right)}{n_{p}}=\sqrt{\frac{\sum_{i^{\prime \prime}=i}^{i^{\prime}}\left(\left\|\boldsymbol{\tau}_{i^{\prime \prime}, k}\right\|_{2}-\overline{\|\tau\|_{2}}\right)^{2}}{i^{\prime}-i}} \frac{\left(i^{\prime}-i\right)}{n_{p}}
$$

\subsection{Step X: Dijkstra's Algorithm}

A Dijkstra's algorithm [33], [34], [35] is used to select the optimal reconfiguration strategy. It searches for the graph in order to compute the shortest path connecting nodes $N_{0}$ and $N_{e}$. The optimal sequence of nodes to be crossed corresponds to the optimal sequence of reconfigurations to be performed to allow the moving platform to follow the whole prescribed path. The optimization can be performed by analyzing different criteria, and consequently different costs associated to the arcs of the graph. The cost of the optimal reconfiguration strategy, $\Phi_{t}$, is equal to the sum of the costs of the arcs connecting the nodes belonging to the shortest path.

\section{Case Study}

The reconfiguration planning method introduced in Section 3 is implemented in the following case study which aims at painting and sandblasting the yellow tubular structure shown in Fig. 10. This structure is $5 \mathrm{~m}$ long, $2 \mathrm{~m}$ wide at its extremities and $1.5 \mathrm{~m}$ wide at its center and lies on the ground. The tubes have a diameter $\phi_{t}$ equal to $10 \mathrm{~cm}$. The RCDPR should paint and sandblast both the inner and the outer faces of the structure. A robotic arm, embarked on the moving platform, aims at manipulating the sandblasting and painting tools. Therefore, the orientation of the moving platform can be kept constant and the $\mathbf{x}_{p}, \mathbf{y}_{p}$ and $\mathbf{z}_{p}$ axes of the moving platform frame $\mathscr{F}_{p}$ are supposed to be parallel to the $\mathbf{x}_{b}, \mathbf{y}_{b}$ and $\mathbf{z}_{b}$ axes of the base frame $\mathscr{F}_{b}$.

\subsection{Problem Description}

\subsubsection{Constant Design Parameters (Step I)}

The RCDPR consists of a moving platform connected to the base by $m=8$ cables, as shown in Fig. 10. The characteristics of the cables of the RCDPR are the following: The Young Modulus $E$ is equal to $100 \mathrm{GPa}$, the cable diameter $\phi_{c}$ is equal to $4 \mathrm{~mm}$, the stiffness coefficient $k_{i}$ is equal to $252 \mathrm{KN} / \mathrm{m}$, and the structural maximum cable 


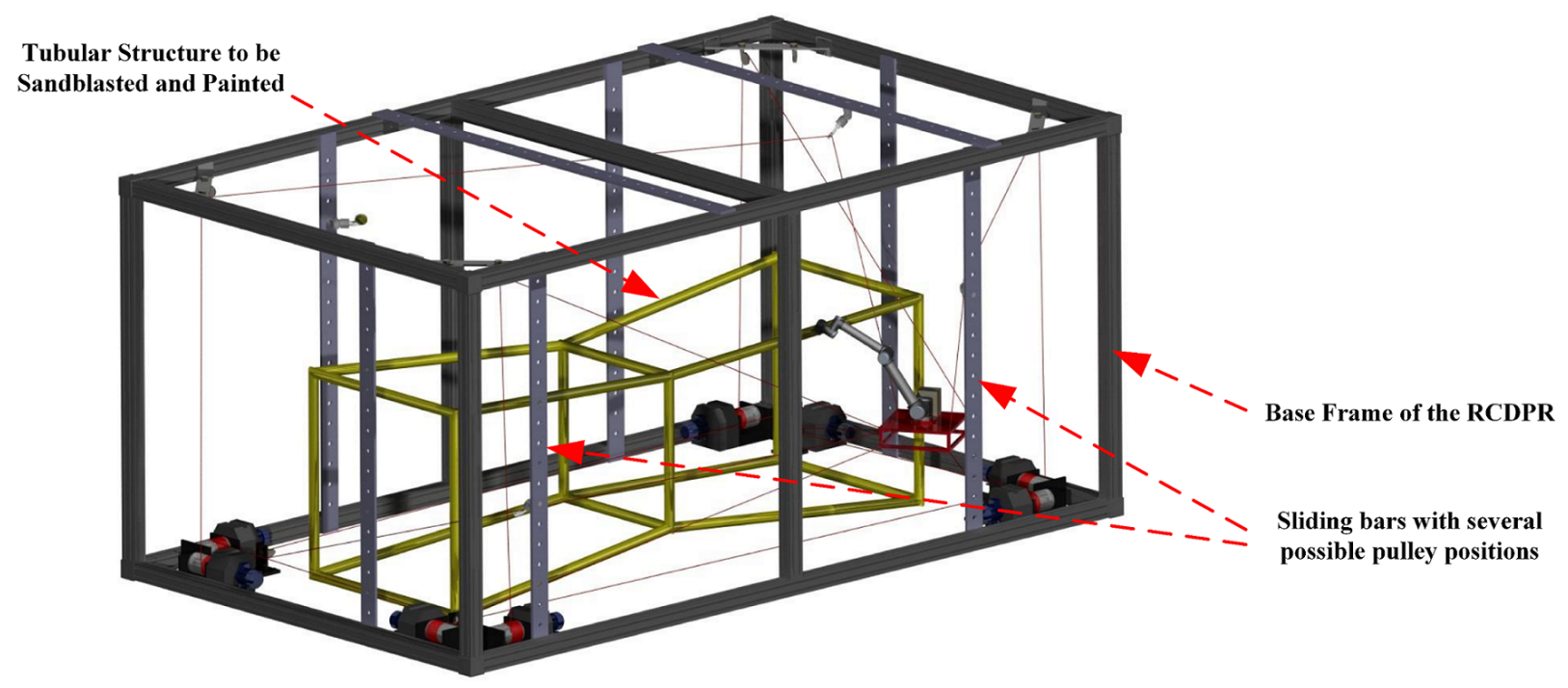

Figure 10: Case study: The yellow tubular structure should be sandblasted and painted by the RCDPR. The RCDPR consists of a moving platform, eight cables and a base with some sliding bars.

tension is equal to $34.95 \mathrm{KN}$. A tension safety coefficient, $k_{s 1}=5$, has been applied in this case study so that $\tau_{\max 1}=6.99 \mathrm{KN}$.

The base length, $l_{s}$, is equal to $7 \mathrm{~m}$, the base width, $w_{s}$, is equal to $4.5 \mathrm{~m}$ and the base height, $h_{s}$, is equal to $4 \mathrm{~m}$. The cables are connected to the base frame through some pulleys. The pulleys can be installed on a predefined grid of locations which are located on ten sliding bars. The sliding bars are installed on the lateral and top sides of the base. Their motion is discretized with a constant step of $25 \mathrm{~cm}$.

The cables are coiled and uncoiled on winches, which are actuated by electric motors. The characteristics of the power system are the following: The nominal torque of the motor, $\tau_{M}$, is equal to $45 \mathrm{Nm}$, the nominal speed, $\omega_{M}$, is equal to $3000 \mathrm{rpm}$, the gearbox transmission ratio, $\rho_{R}$, is equal to 10 , the diameter of the winches, $\rho_{W}$, is equal to $15 \mathrm{~cm}$. The cable tension limit $\tau_{\max }$ is equal to $\tau_{\max }=\min \left\{\tau_{\max 1}, \tau_{\max 2}\right\}=6000 N$. Considering that $\tau_{\max 2}=\frac{2}{\phi_{w}} \rho_{R} \tau_{M}=6000 \mathrm{~N}, \tau_{\max }=\tau_{\max 2}$ since the tension that can be provided to the cables is lower than $\tau_{\max 1}$.

The moving platform is represented in Fig. 11. Its width, $w_{p}$, is equal to $20 \mathrm{~cm}$, its length, $l_{p}$, is equal to $20 \mathrm{~cm}$ and its height is equal to $25 \mathrm{~cm}$. The Cartesian coordinates of points $B_{i}, i=1, \ldots, 8$, are expressed in frame $\mathscr{F}_{p}$ as 
follows:

$$
\begin{aligned}
\mathbf{b}_{1}^{p} & =\left[-w_{p} / 2-c_{0},-l_{p} / 2,-h_{p} / 2\right] \\
\mathbf{b}_{2}^{p} & =\left[-w_{p} / 2,-l_{p} / 2-c_{0}, h_{p} / 2\right] \\
\mathbf{b}_{3}^{p} & =\left[-w_{p} / 2-c_{0}, l_{p} / 2,-h_{p} / 2\right] \\
\mathbf{b}_{4}^{p} & =\left[-w_{p} / 2, l_{p} / 2+c_{0}, h_{p} / 2\right] \\
\mathbf{b}_{5}^{p} & =\left[w_{p} / 2+c_{0}, l_{p} / 2,-h_{p} / 2\right] \\
\mathbf{b}_{6}^{p} & =\left[w_{p} / 2, l_{p} / 2+c_{0}, h_{p} / 2\right] \\
\mathbf{b}_{7}^{p} & =\left[w_{p} / 2+c_{0},-l_{p} / 2,-h_{p} / 2\right] \\
\mathbf{b}_{8}^{p} & =\left[w_{p} / 2,-l_{p} / 2-c_{0}, h_{p} / 2\right]
\end{aligned}
$$

where $c_{0}$ is an offset used to avoid both the collisions between the cables and the parallel singularities of the RCDPR (the wrench matrix $\mathrm{W}$ has not full rank). The offset helps to prevent this situation by introducing an asymmetry between the layout of the cable exit points and the layout of the cable connection points on the platform. In this case study, the offset is equal to $3.5 \mathrm{~cm}$.

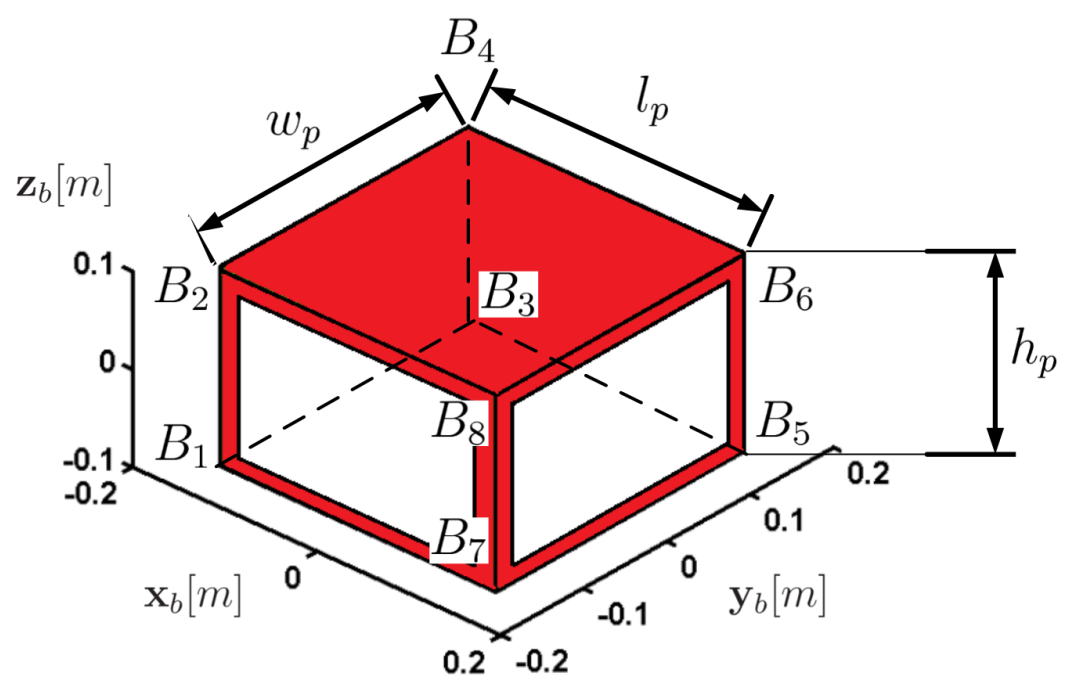

Figure 11: Moving platform.

\subsubsection{RCDPR Layout Parametrization and Configuration Set (Steps II and III)}

Two different layouts and parametrizations of the RCDPR are used in order to cover the inner and the outer areas of the tubular structure. In the layout chosen for the outer faces of the structure, the exit points, $A_{i}, i=1, \ldots, 8$, are located at the vertices of a parallelepiped. The Cartesian coordinates of points $A_{i}, i=1, \ldots, 8$ are parametrized by 
means of four parameters $v_{1}, v_{2}, v_{3}$ and $v_{4}$ as shown in Fig. 12:

$$
\begin{aligned}
\mathbf{a}_{1}^{b} & =\left[\begin{array}{lll}
v_{1} & -l_{s} / 2 & v_{4}
\end{array}\right]^{\mathrm{T}} & \mathbf{a}_{2}^{b} & =\left[\begin{array}{lll}
v_{1} & -l_{s} / 2 & v_{2}
\end{array}\right]^{\mathrm{T}} \\
\mathbf{a}_{3}^{b} & =\left[\begin{array}{lll}
v_{1} & l_{s} / 2 & v_{4}
\end{array}\right]^{\mathrm{T}} & \mathbf{a}_{4}^{b} & =\left[\begin{array}{lll}
v_{1} & l_{s} / 2 & v_{2}
\end{array}\right]^{\mathrm{T}} \\
\mathbf{a}_{5}^{b} & =\left[\begin{array}{lll}
v_{3} & l_{s} / 2 & v_{4}
\end{array}\right]^{\mathrm{T}} & \mathbf{a}_{6}^{b} & =\left[\begin{array}{lll}
v_{3} & l_{s} / 2 & v_{2}
\end{array}\right]^{\mathrm{T}} \\
\mathbf{a}_{7}^{b} & =\left[\begin{array}{lll}
v_{3} & -l_{s} / 2 & v_{4}
\end{array}\right]^{\mathrm{T}} & \mathbf{a}_{8}^{b} & =\left[\begin{array}{lll}
v_{3} & -l_{s} / 2 & v_{2}
\end{array}\right]^{\mathrm{T}}
\end{aligned}
$$

The four parameters can take the following values:

$$
\begin{aligned}
& {[v]_{1}=\{-2.10,-1.85,-1.60,-1.35,-1.10,1.10,1.35,1.60,1.85,2.10\}} \\
& {[v]_{2}=\{0.10,0.35,0.60,0.85,1.10,1.90,2.15,2.40,2.65,2.90,3.15,3.40\}} \\
& {[v]_{3}=\{-2.10,-1.85,-1.60,-1.35,-1.10,1.10,1.35,1.60,1.85,2.10\}} \\
& {[v]_{4}=\{0.10,0.35,0.60,0.85,1.10,1.90,2.15,2.40,2.65,2.90,3.15,3.40\}}
\end{aligned}
$$

Those values for parameters $v_{1}, v_{2}, v_{3}$ and $v_{4}$ lead to $n_{C}=14400$ possible configurations of the RCDPR.
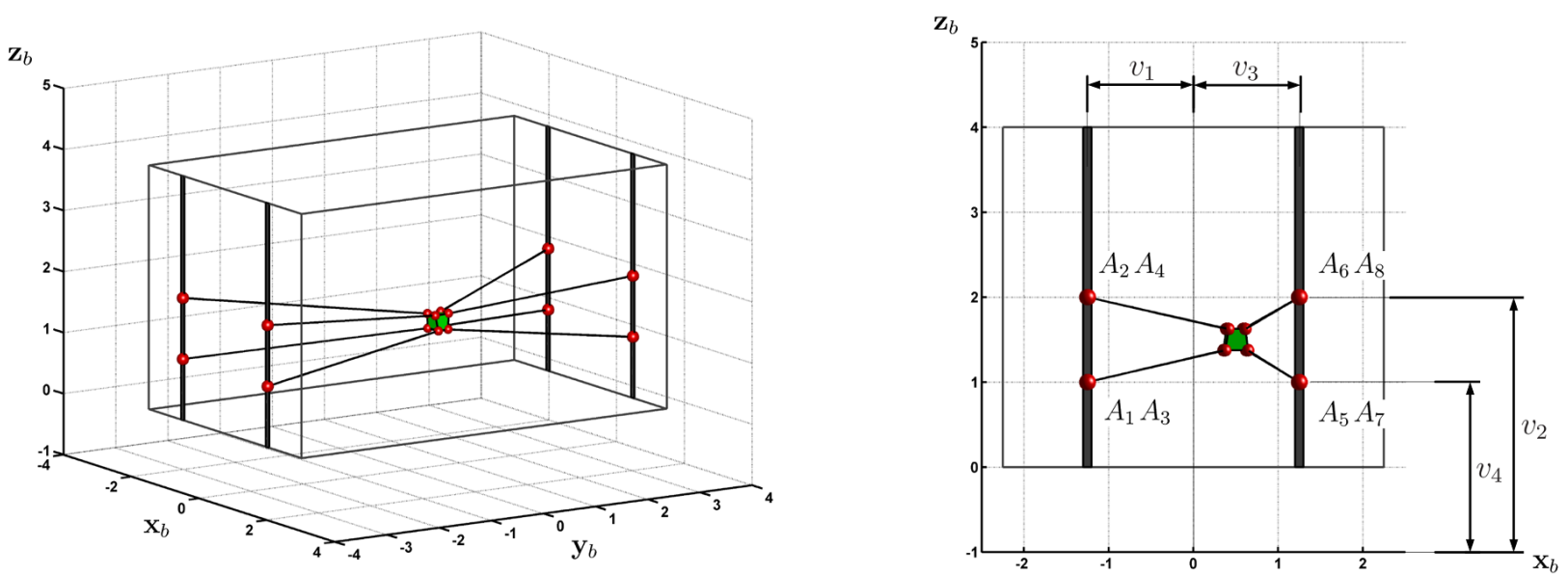

Figure 12: Parametrization of the RCDPR while operating on the outer faces of the tubular structure.

The exit point layout used to cover the inner faces of the structure is a parallelepiped too, as shown in Fig. 13. However, considering the symmetry of the task, the exit points $A_{i}, i=1, \ldots, 8$ are located symmetrically with respect to plane $\mathbf{y}_{b} O \mathbf{z}_{b}$. As a consequence, the three parameters $w_{1}, w_{2}$ and $w_{3}$ are considered to be sufficient to define the 

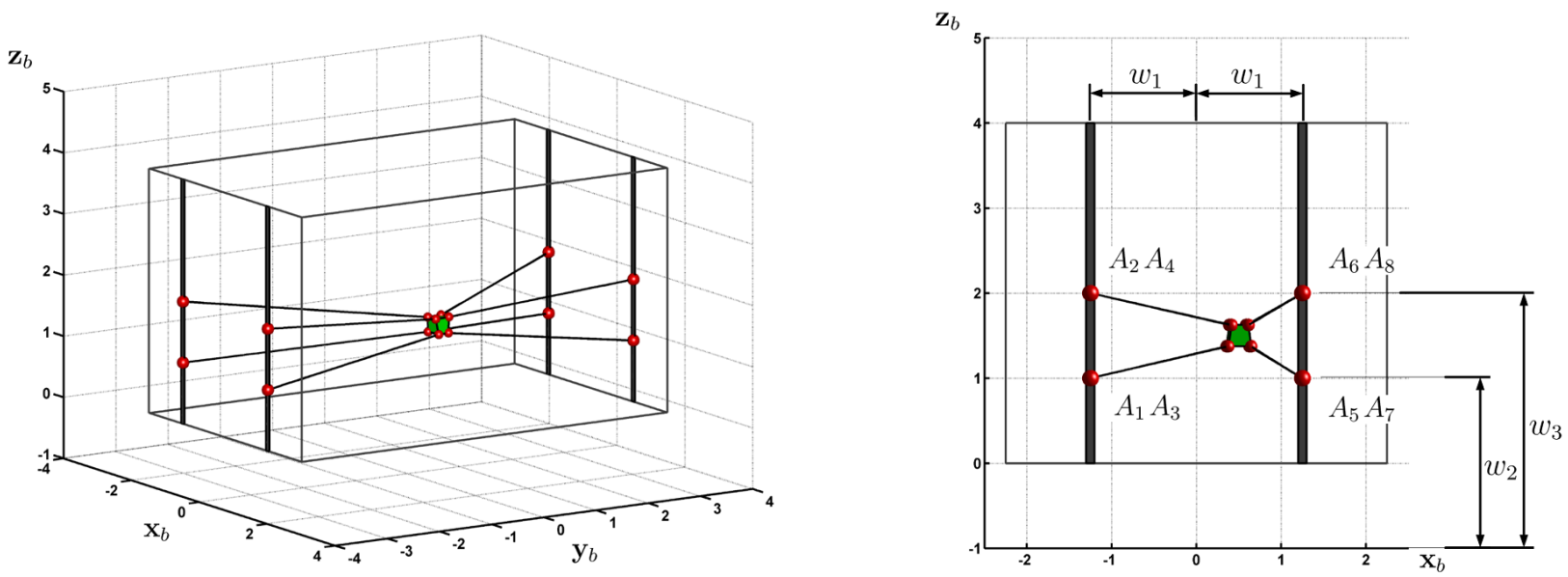

Figure 13: Parametrization of the RCDPR while operating on the inner faces of the tubular structure.

Cartesian coordinates of points $A_{i}$ :

$$
\begin{aligned}
\mathbf{a}_{1}^{b} & =\left[\begin{array}{lll}
-w_{1} & -l_{s} / 2 & w_{2}
\end{array}\right]^{\mathrm{T}} & \mathbf{a}_{2}^{b} & =\left[\begin{array}{lll}
-w_{1} & -l_{s} / 2 & w_{3}
\end{array}\right]^{\mathrm{T}} \\
\mathbf{a}_{3}^{b} & =\left[\begin{array}{lll}
-w_{1} & l_{s} / 2 & w_{2}
\end{array}\right]^{\mathrm{T}} & \mathbf{a}_{4}^{b} & =\left[\begin{array}{lll}
-w_{1} & l_{s} / 2 & w_{3}
\end{array}\right]^{\mathrm{T}} \\
\mathbf{a}_{5}^{b} & =\left[\begin{array}{lll}
w_{1} & l_{s} / 2 & w_{2}
\end{array}\right]^{\mathrm{T}} & \mathbf{a}_{6}^{b} & =\left[\begin{array}{lll}
w_{1} & l_{s} / 2 & w_{3}
\end{array}\right]^{\mathrm{T}} \\
\mathbf{a}_{7}^{b} & =\left[\begin{array}{lll}
w_{1} & -l_{s} / 2 & w_{2}
\end{array}\right]^{\mathrm{T}} & \mathbf{a}_{8}^{b} & =\left[\begin{array}{lll}
w_{1} & -l_{s} / 2 & w_{3}
\end{array}\right]^{\mathrm{T}}
\end{aligned}
$$

Parameters $w_{1}, w_{2}$ and $w_{3}$ can take the following values:

$$
\begin{aligned}
& {[w]_{1}=\{0,0.25,0.5,0.75,1.0,1.25,1.5,1.75,2.0,2.25\}} \\
& {[w]_{2}=\{0.25,0.5,0.75,1.0,1.25,1.5,1.75,2.0,2.25\}} \\
& {[w]_{3}=\{0.25,0.5,0.75,1.0,1.25,1.5,1.75,2.0,2.25\}}
\end{aligned}
$$

Those values for parameters $w_{1}, w_{2}$ and $w_{3}$ lead to $n_{C}=810$ possible configurations of the RCDPR.

\subsubsection{Task and Environment (Step IV)}

The weight of the moving platform and the embarked tools is equal to $31 \mathrm{Kg}$. The forces transmitted by the painting and sandblasting tools to the moving platform are bounded as follows:

$$
\begin{gathered}
-30 \mathrm{~N} \leq f_{x}, f_{y} \leq 30 \mathrm{~N} \\
f_{z}=-310 \mathrm{~N}
\end{gathered}
$$

The moments $m_{x}, m_{y}$ and $m_{z}$ about axes $\mathbf{x}_{b}, \mathbf{y}_{b}$ and $\mathbf{z}_{b}$ are neglected: $m_{x}, m_{y}, m_{z}=0$. 
In order to complete the operations, the CoM of the moving platform should follow the profile of the tubular structure with an offset $d_{t}=80 \mathrm{~cm}$ with respect to the central axes of the tubes. Figure 14 shows the red segments and the green via points that the path prescribed to the moving platform should follow. According to the procedure described in Sec. 3.4, an Eulerian path, $\mathscr{P}_{\text {out }}$, and an Eulerian cycle, $\mathscr{P}_{\text {in }}$, covering the outer and inner faces of the tubular structure, respectively, can be defined. $\mathscr{P}_{\text {out }}$ is discretized into 450 points and $\mathscr{P}_{\text {in }}$ is discretized into 500 points. The Eulerian path, $\mathscr{P}_{\text {out }}$, is illustrated in Fig. 15. The moving-platform should go through the green via points while respecting a prescribed order. For instance, the moving-platform should follow the Eulerian path, $\mathscr{P}_{\text {out }}$, while passing through 23 points in sequence, namely from point $P_{1}$ to point $P_{23}$, as shown in Fig. 15. Note that points $P_{1}$, $P_{5}, P_{11}$ and $P_{23}$ are coincident and are named $P_{1,5,11,23}$.
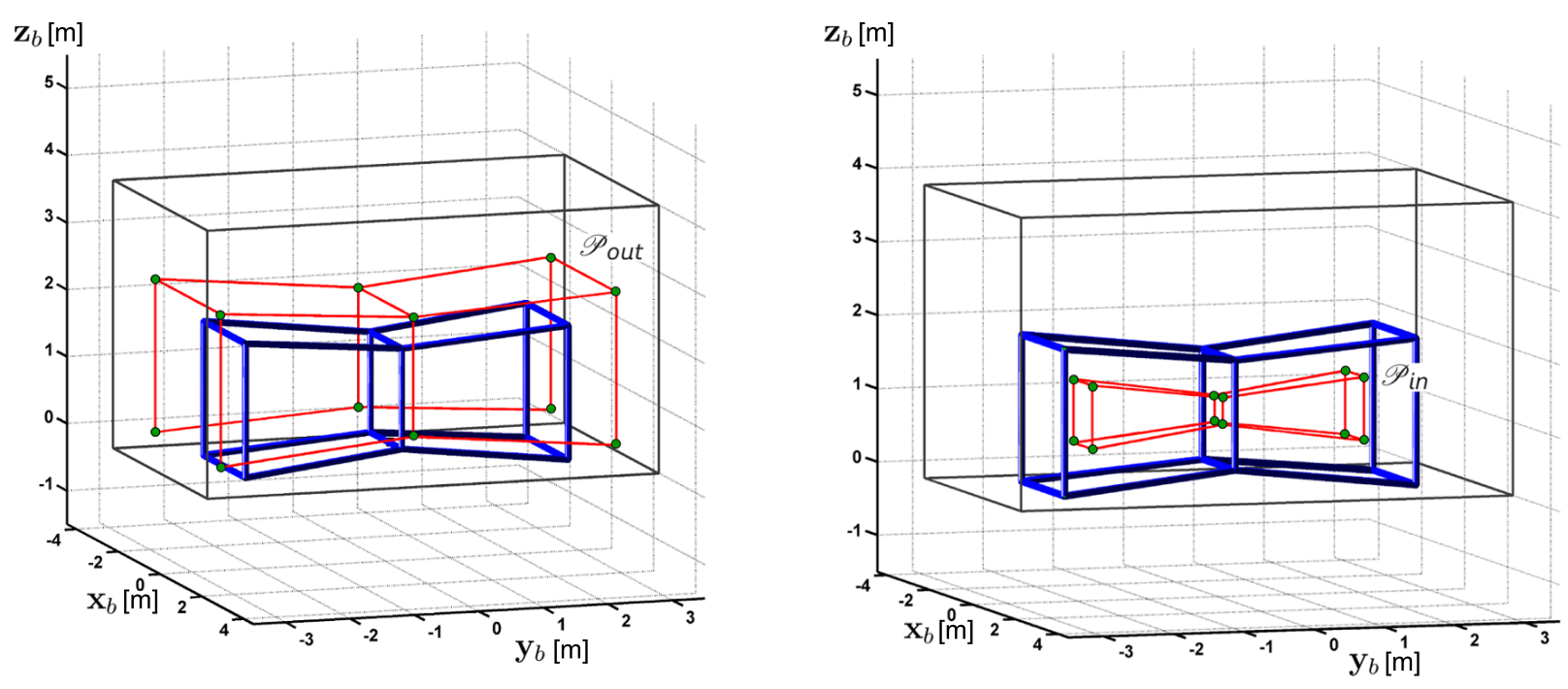

Figure 14: Sets of red segments and green via points to be covered by the platform CoM. 


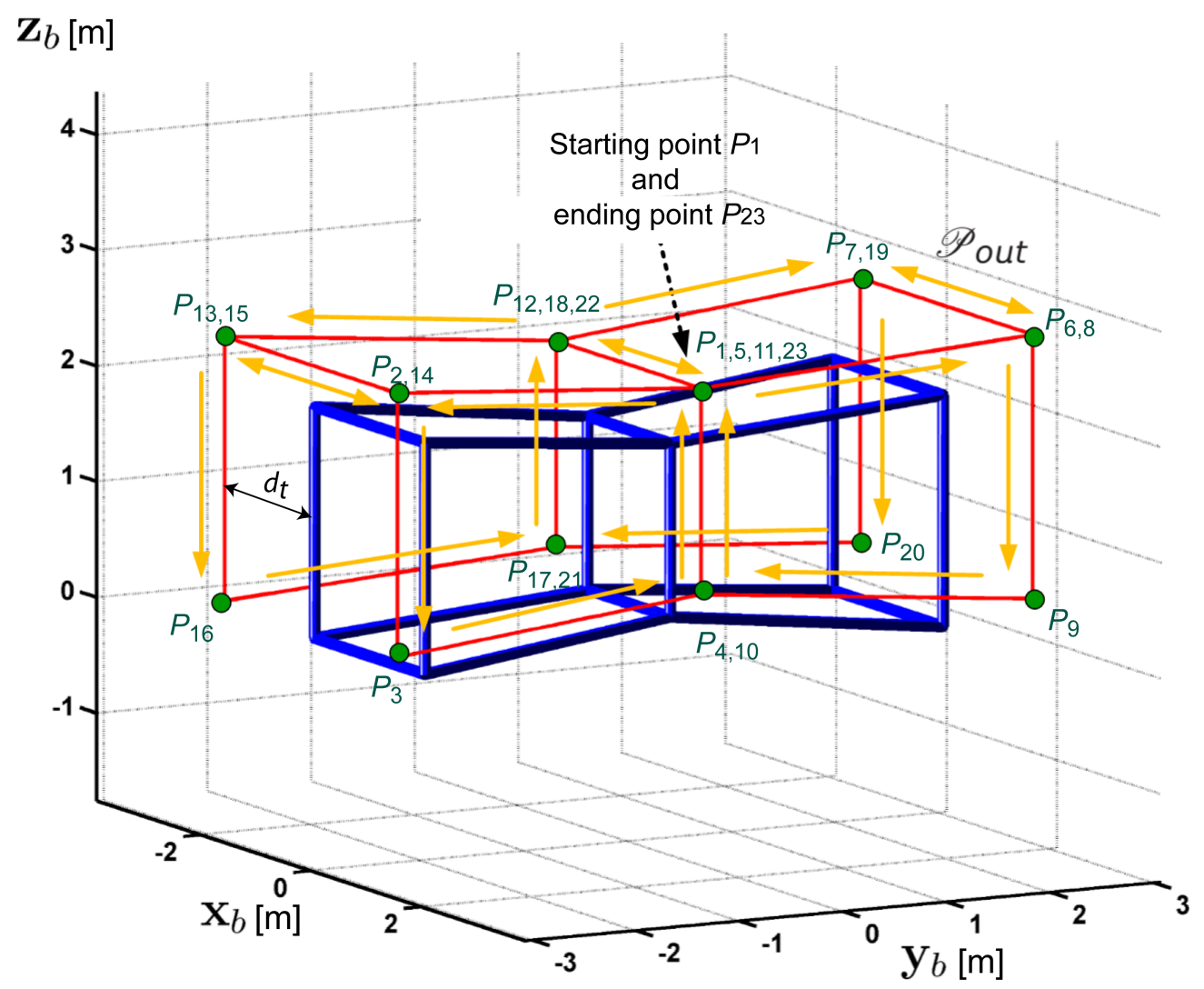

Figure 15: Eulerian path $\mathscr{P}_{\text {out }}$ to be followed by the platform CoM when moving on the outer faces of the tubular structure. 


\subsection{Results for the Outer Faces of the Tubular Structure}

\subsubsection{Feasibility Map and Configuration Selection (Steps VI and VII)}

The $n_{\mathcal{C}}=14400$ possible configurations of the RCDPR obtained in the previous section are analyzed in order to identify the segments of $\mathscr{P}_{\text {out }}$ that can be covered by each configuration. Then, the constraints introduced in Sec. 3.5 are considered leading to trace a feasibility map composed of 14400 lines, each line corresponding to one RCDPR configuration.

The problem turns out to be fairly complex because of the large number of lines in the feasibility map. In order to reduce the problem complexity, the configuration selection approach explained in Sec. 3.7 is used to reduce the number of configurations to be considered with the following thresholds: $h_{1}=20$ and $h_{2}=0.25$. As a result, $n_{d}=76$ dominant configurations are obtained among $n_{C^{\prime}}=1186$ filtered configurations. It is noteworthy that the minimum number of configurations required to complete the task among the 76 dominant configurations is equal to $n_{m}=3$.

Moreover, 784 triplets of dominant configurations can be used to cover the prescribed path $\mathscr{P}_{\text {out }}$ completely. Note that 57 configurations can be considered among the 76 dominant ones to generate the 784 triplets. The 19 remaining dominant configurations necessarily lead to a combination of more than three dominant configurations to cover the whole prescribed path.

\subsubsection{Graph Building and Cost Functions (Steps VIII and IX)}

Figure 16 illustrates the graph obtained from the selected 57 dominant configurations. The graph is composed of 452 nodes and 10752 arcs. Due to the size of this graph, only a partial representation has been provided in Fig. 16.

The corresponding reconfiguration planning problem has been solved using a Dijkstra's algorithm with respect to each cost function, $\mu_{i}=i=1, \ldots, 5$, proposed in Sec. 3.9.

The optimal solutions associated to each of the five cost functions are represented on the feasibility map shown in Fig. 17. For the sake of clarity, only the 10 configurations, $C_{i}^{*}, i=1, \ldots, 10$, composing the five different optimal solutions are displayed on the feasibility map. Table 1 gives the design parameters corresponding to those 10 configurations.

\subsubsection{Results Analysis}

From Fig. 17, it is apparent that configurations $C_{[1-4]}^{*}$ have the same feasibility map, as well as configurations $C_{[6-10]}^{*}$. Indeed, from Def. 4, two dominant configurations, generated by different design parameters, can share the same feasibility map. Note that, different configurations can present the same feasibility map but generally provide different performances. For instance, configuration $C_{10}^{*}$ is better in terms of cable tensions than configurations $C_{[6-9]}^{*}$.

While using $\mu_{1}$ as the cost function (arc cost in Dijkstra's algorithm), the number of required reconfigurations to complete the task is minimized. Five reconfigurations turn out to be sufficient to complete the task. $C_{10}^{*}$ is the starting configuration. 


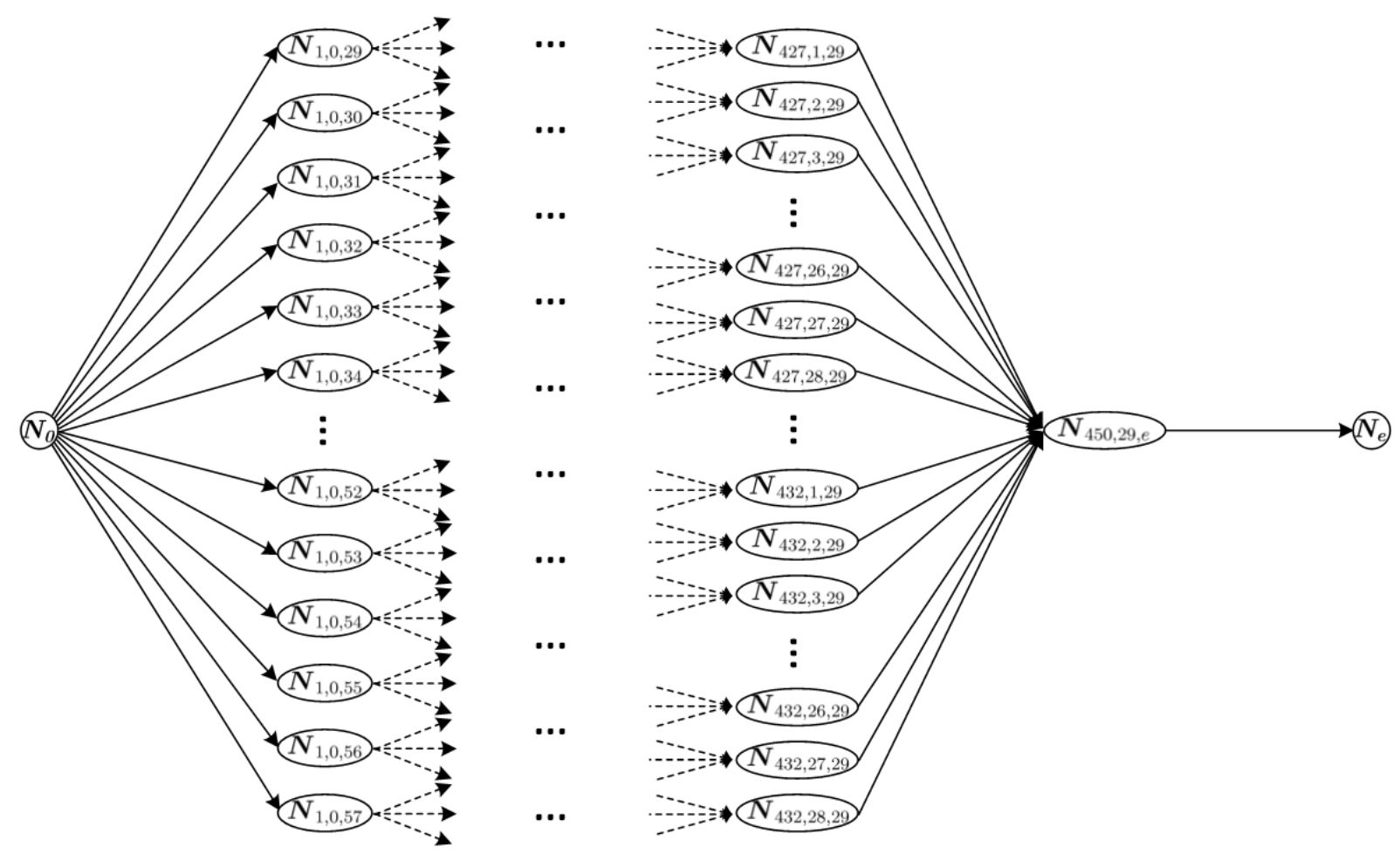

Figure 16: Partial representation of the reconfiguration graph for the case study. The graph is composed of 452 nodes and 10752 arcs.

Table 1: Optimal configurations for the spatial RCDPR.

\begin{tabular}{c|cccc}
\hline Configuration & $v_{1}$ & $v_{2}$ & $v_{3}$ & $v_{4}$ \\
\hline$C_{1}^{*}$ & -2.1 & 0.1 & -1.1 & 3.4 \\
$C_{2}^{*}$ & -1.85 & 0.1 & -1.1 & 3.4 \\
$C_{3}^{*}$ & -1.85 & 0.35 & -1.1 & 3.4 \\
$C_{4}^{*}$ & -1.85 & 0.85 & -1.1 & 3.4 \\
$C_{5}^{*}$ & -1.85 & 2.4 & 1.85 & 3.4 \\
$C_{6}^{*}$ & 1.1 & 0.1 & 1.85 & 2.65 \\
$C_{7}^{*}$ & 1.1 & 0.1 & 1.85 & 3.4 \\
$C_{8}^{*}$ & 1.1 & 0.1 & 2.1 & 3.4 \\
$C_{9}^{*}$ & 1.1 & 0.6 & 1.85 & 3.4 \\
$C_{10}^{*}$ & 1.1 & 0.85 & 1.85 & 3.4 \\
\hline
\end{tabular}



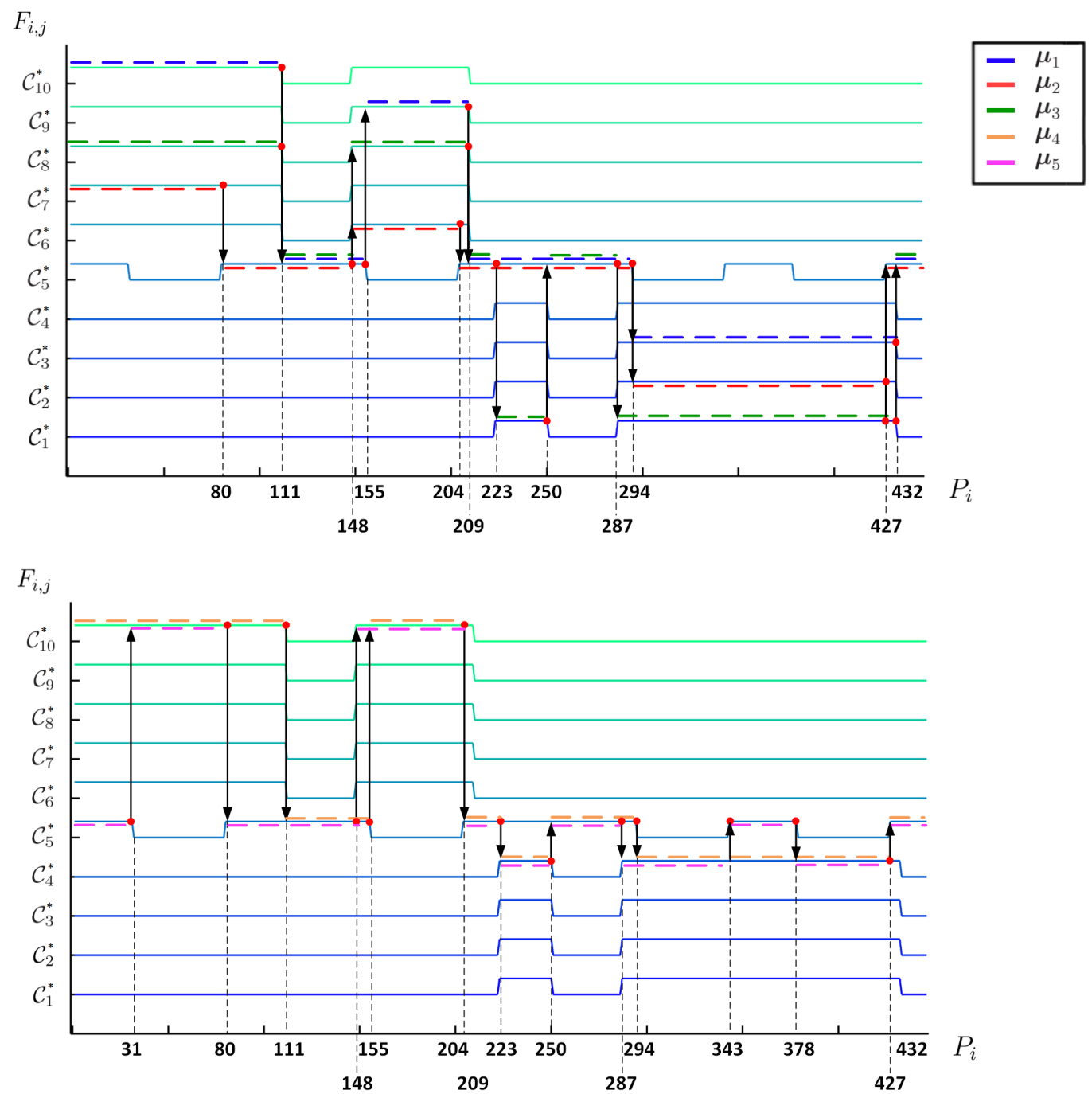

Figure 17: Feasibility map and optimal solutions for the spatial RCDPR. 
Table 2: Optimal reconfiguration planning and the associated cost functions for the outer faces of the tubular structure. For each reconfiguration strategy, the optimized cost function is surrounded by a red box.

\begin{tabular}{l|rrrrrr}
\hline \multirow{2}{*}{ Optimal Reconfiguration Planning } & \multicolumn{5}{|c}{ Cost of the reconfiguration strategy } \\
\cline { 2 - 6 } & $\mu_{1}[]$ & $\mu_{2}[\mathrm{~N}]$ & $\mu_{3}[\mathrm{~mm}]$ & $\mu_{4}[\mathrm{~N}]$ & $\mu_{5}[\mathrm{~N}]$ \\
\hline Optimal planning w.r.t. $\mu_{1}$ & 30 & 234.8 & 0.208 & 578.1 & 233.9 \\
Optimal planning w.r.t. $\mu_{2}$ & 34 & 281.3 & 0.217 & 615.0 & 232.8 \\
Optimal planning w.r.t. $\mu_{3}$ & 56 & 260.4 & 0.132 & 751.2 & 504.5 \\
Optimal planning w.r.t. $\mu_{4}$ & 42 & 238.9 & 0.205 & 542.3 & 203.5 \\
Optimal planning w.r.t. $\mu_{5}$ & 60 & 135.0 & 0.227 & 561.8 & 158.5 \\
\hline
\end{tabular}

At the $11^{\text {th }}$ point, $P_{11}$, of the discretized path $\mathscr{P}_{\text {out }}$, all the constraints of the optimization problem at hand are not satisfied anymore for configuration $C_{10}^{*}$. As a consequence, the RCDPR switches from configuration $C_{10}^{*}$ to configuration $C_{5}^{*}$. During the reconfiguration, all the exit points have to be displaced, except points $A_{5}$ and $A_{7}$. A NFT is then encountered at $P_{155}$ and the RCDPR switches to configuration $C_{9}^{*}$. During this reconfiguration, exit points $A_{5}$ and $A_{7}$ are not displaced. The RCDPR meets another NFT at $P_{209}$ and six of the exit points are displaced. Between points $P_{209}$ and $P_{294}$, the RCDPR remains in configuration $C_{5}^{*}$. Two other reconfigurations are necessary to reach the end of $\mathscr{P}_{\text {out }}$, as shown in Fig. 17. Table 2 gives the optimal value of each cost function $\mu_{i}=i=1, \ldots, 5$, obtained with Dijkstra's algorithm.

The optimization performed with respect to $\mu_{2}$ leads to five reconfigurations, as well, while maximizing the capacity margin of the RCDPR. $C_{7}^{*}$ is the starting configuration. The first reconfiguration occurs at point $P_{80}$, in presence of a PFT. The RCDPR switches to configuration $C_{5}^{*}$. The portions of $\mathscr{P}_{\text {out }}$ between $P_{80}$ and $P_{148}$ and between $P_{204}$ and $P_{294}$ cover the upper face of the tubular structure. Configuration $C_{5}^{*}$ is assigned to these portions of $\mathscr{P}_{\text {out }}$ since it improves the capacity margin. In fact, it should be noted that $C_{5}^{*}$ is a suspended configuration and suspended configurations usually provide a higher capacity margin than fully constrained ones. The fully constrained configurations $C_{6}^{*}$ and $C_{2}^{*}$ are used on the lateral faces of the tubular structure. The RCDPR switches to these configuration when a PFT or a NFT is encountered.

The optimization performed while considering $\mu_{3}$ minimizes the use of the suspended configuration $C_{5}^{*}$. In order to maintain a static equilibrium, a fully constrained configuration usually requires higher cable tensions than a suspended configuration. Larger cable tensions allow us to increase the RCDPR stiffness, reducing the positioning errors. The positioning error optimization leads to configuration $C_{8}^{*}$ at the beginning of $\mathscr{P}_{\text {out }}$. When a NFT is encountered, at $P_{111}$, the RCDPR switches to configuration $C_{5}^{*}$, coming back to configuration $C_{8}^{*}$ when a PFT is encountered at $P_{148}$. Following the same principle, the RCDPR uses configuration $C_{5}^{*}$ only when the fully constrained configurations become infeasible. It goes back to configurations $C_{1}^{*}$ or $C_{8}^{*}$, as soon as possible, when following configuration $C_{5}^{*}$ a PFT is encountered. It is noteworthy that, despite the high number of reconfigurations, only three configurations $\left(C_{1}^{*}\right.$, $C_{5}^{*}$ and $C_{8}^{*}$ ) are required to complete the task. The fully constrained ones, $C_{1}^{*}$ and $C_{8}^{*}$, are symmetric with respect to 


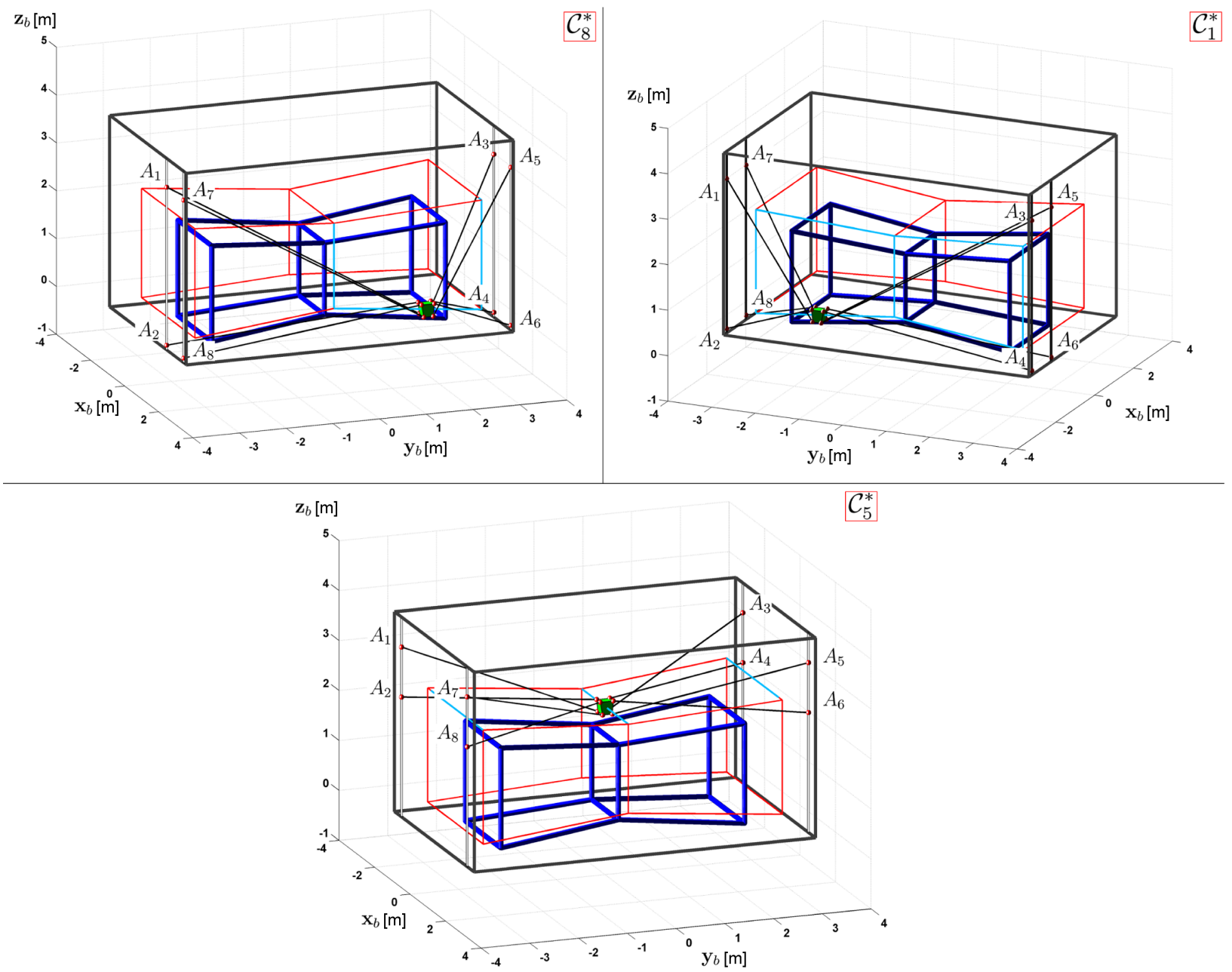

Figure 18: Configurations $C_{1}^{*}, C_{5}^{*}$ and $C_{8}^{*}$, composing the optimal reconfiguration planning with respect to cost function $\mu_{3}$. 
the plane $\mathbf{y}_{b} O \mathbf{z}_{b}$, as shown in Fig. 18. Furthermore, it has to be noticed that, despite an improvement of the platform positioning error, the optimal reconfiguration planning increases the cable tension average to $751.2 \mathrm{~N}$ and the average of the cable tension variations to $504.5 \mathrm{~N}$.

The optimizations performed while considering $\mu_{4}$ and $\mu_{5}$ as cost functions are similar: The two solutions assign the same configurations to a large portion of $\mathscr{P}_{\text {out }}$. In both the optimization problems, the configurations taking part to the optimal solutions are $C_{4}^{*}, C_{5}^{*}$ and $C_{10}^{*}$. The optimal reconfiguration planning with respect to $\mu_{4}$ requires the RCDPR to perform seven reconfigurations and displaces 42 exit points. The number of reconfigurations is equal to ten when the cable tension variation (represented by $\mu_{5}$ ) is optimized, and the number of exit point displacements is equal to 60 . Figure 19 illustrates the optimal reconfiguration scheme of the RCDPR obtained for cost function $\mu_{5}$.
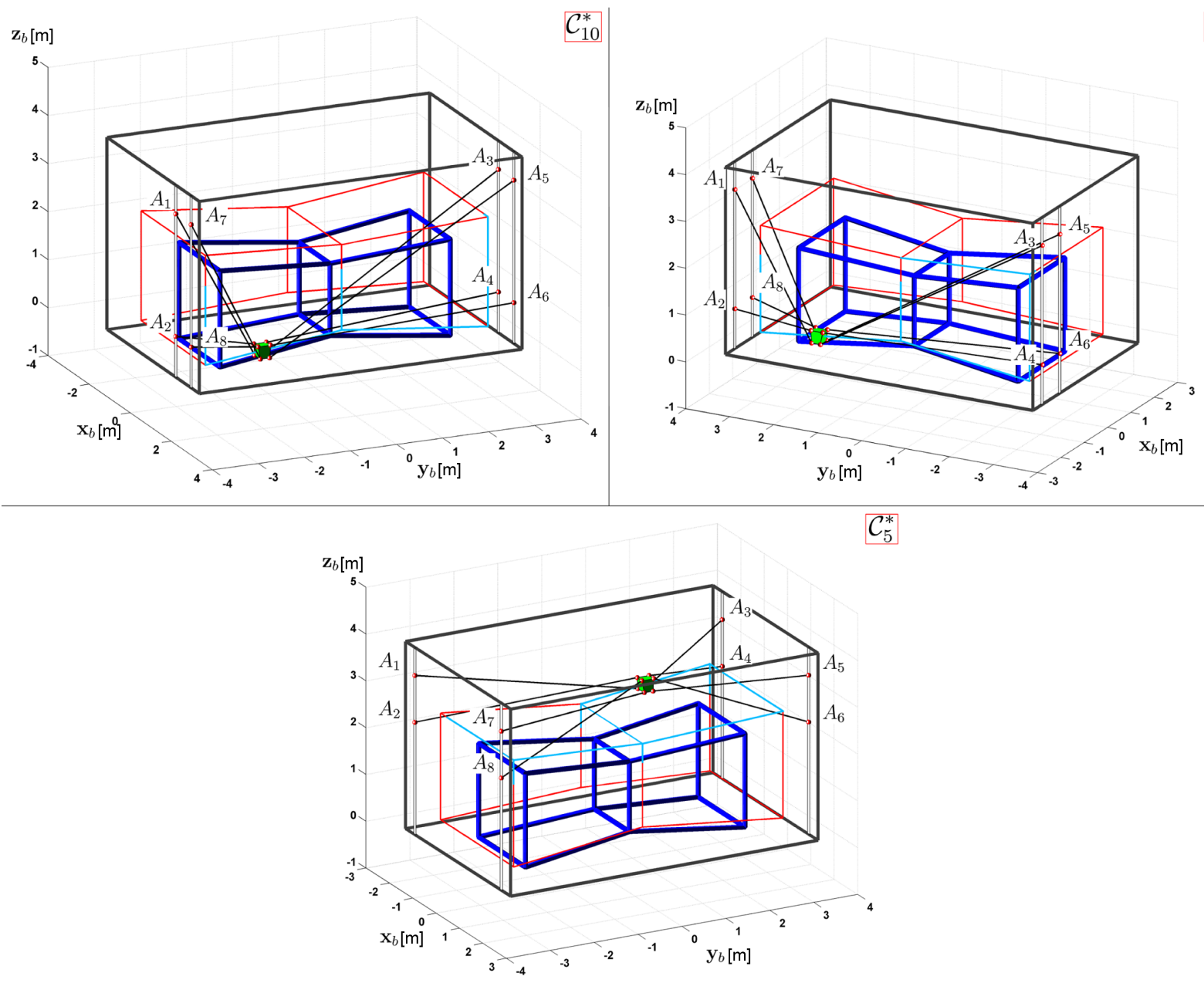

Figure 19: Configurations $C_{4}^{*}, C_{5}^{*}$ and $C_{10}^{*}$, composing the optimal reconfiguration planning with respect to cost function $\mu_{5}$. 
Table 3: Optimal configurations and the associated cost functions for the inner faces of the tubular structure. For each configuration, the optimal cost functions are surrounded by red boxes.

\begin{tabular}{ll|lrrr}
\hline Optimal Configurations & & $\mu_{2}[\mathrm{~N}]$ & $\mu_{3}[\mu \mathrm{m}]$ & $\mu_{4}[\mathrm{~N}]$ & $\mu_{5}[\mathrm{~N}]$ \\
\hline$w_{1}=0.75 \mathrm{~m}, w_{2}=2 \mathrm{~m}, w_{3}=1.25 \mathrm{~m}$ & (See Fig. 20) & 526.31 & 157 & 841.99 & \multicolumn{1}{|c}{365.67} \\
$w_{1}=0.75 \mathrm{~m}, w_{2}=2 \mathrm{~m}, w_{3}=0.25 \mathrm{~m}$ & (See Fig. 21) & 166.67 & 331 & 625.81 & 205.69 \\
\hline
\end{tabular}

\subsection{Results for the Inner Faces of the Tubular Structure}

According to Sec. 4.1.2, mixing the design parameters in $[w]_{1},[w]_{2}$ and $[w]_{3}, n_{C}=810$ configurations are obtained. Performing the constraint analysis, a feasibility map has been built and simplified according to the configuration selection procedure described in Sec. 3.7. The thresholds $h_{1}$ and $h_{2}$ have been initialized with the same values used to compute the optimal reconfiguration strategy for the outer faces of the tubular structure.

The analysis of the feasibility map leads to $n_{d}=14$ dominant configurations. Each of these configurations allows the moving platform to cover entirely the path $\mathscr{P}_{\text {in }}$ without any reconfiguration of the robot. Hence, the task can be performed by selecting only one of the dominant configurations. Among those configurations, two of them lead to some optimal solutions.

The configuration whose design parameters $w_{1}, w_{2}$ and $w_{3}$ are equal to $0.75 \mathrm{~m}, 2 \mathrm{~m}$ and $0.25 \mathrm{~m}$, respectively, minimizes the cost functions $\mu_{2}$ and $\mu_{3}$, according to the values given in Tab. 3. According to this configuration, the RCDPR is fully constrained, as shown in Fig. 20. The cables of the RCDPR are crossing (but without any cable-cable collision), improving the RCDPR precision and stiffness.

The configuration whose design parameters $w_{1}, w_{2}$ and $w_{3}$ are equal to $0.75 \mathrm{~m}, 2 \mathrm{~m}$ and $1.25 \mathrm{~m}$, respectively, is shown in Fig. 21. The RCDPR is suspended above the prescribed trajectory. Being suspended, the cable tensions are minimized. This configuration optimizes the cost functions $\mu_{4}$ and $\mu_{5}$. The optimal values are collected in Tab. 3 .

\subsection{Computational Time}

The simulation has been performed on an Intel(R) Core(TM) i7-3630QM CPU @ 2.40 Ghz, with 8 GB of RAM. The results collected in Tab. 4 show that Step III (RCDPR Configuration Set) and Step X (Dijkstra's Algorithm), presented in Sec. 3, are performed in less than $0.02 \mathrm{~s}$. On the contrary, Step VI (Feasibility Map), which involves the constraint analysis of a large number of configurations, required 39 hours of computations. Step VII (Configuration Selection) and Step VIII (Graph Building) required $10.1 \mathrm{~s}$ and 8.2 minutes of computation, respectively. The low computational effort required to perform Step VIII is due to the configuration selection performed at Step VII. Building the graph using the full set of configurations generated at Step III would require several days of intensive computations. The result would lead to a wide graph whose analysis may be prohibitive. 


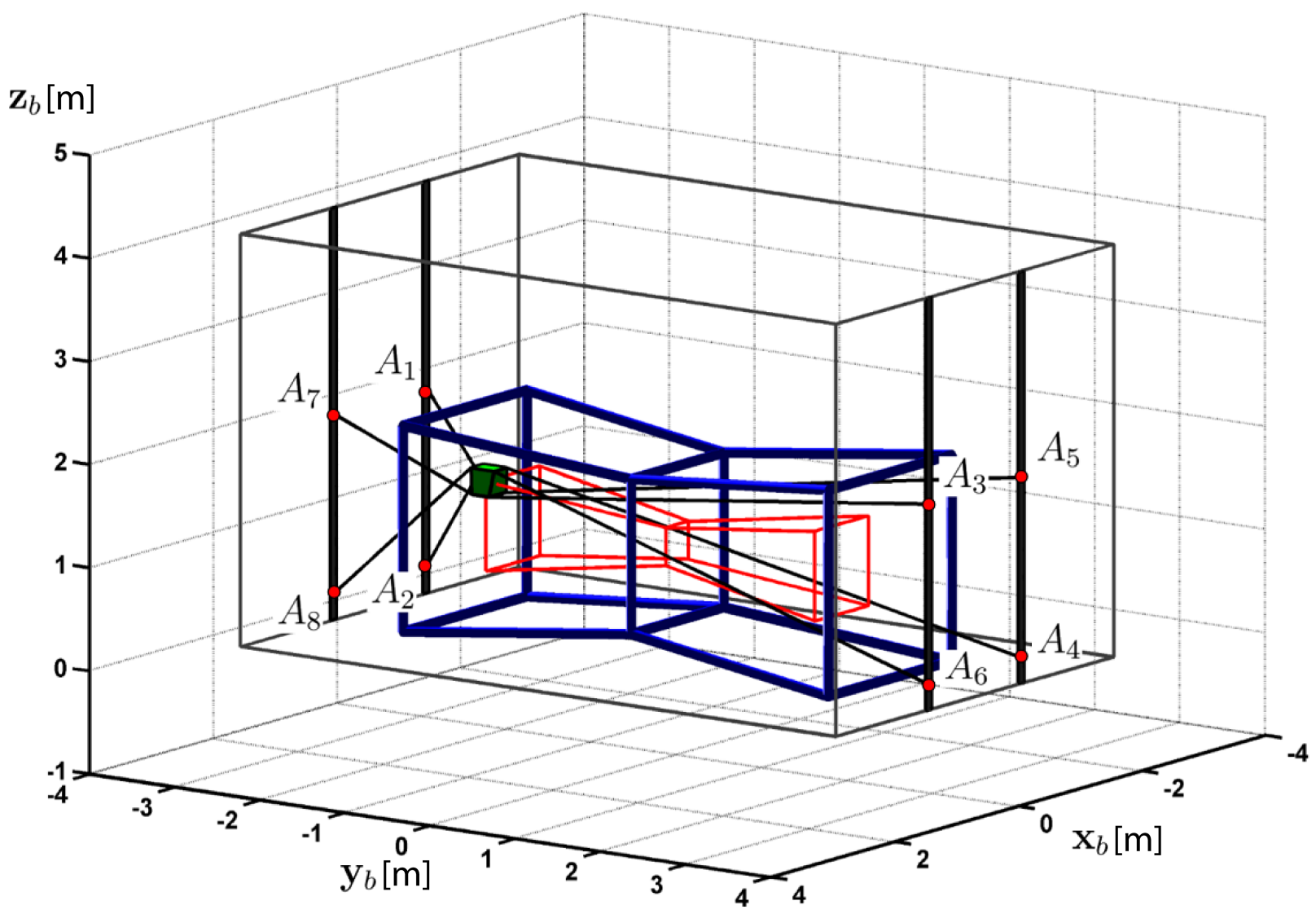

Figure 20: RCDPR configuration, with $w_{1}=0.75 \mathrm{~m}, w_{2}=2 \mathrm{~m}$ and $w_{3}=0.25 \mathrm{~m}$, which optimizes $\mu_{2}$ and $\mu_{3}$ for the inner prescribed path $\mathscr{P}_{\text {in }}$

Table 4: Analysis of the computational time and number of operations for the case study of Sec. 4 .

\begin{tabular}{l|ccc}
\hline Algorithm Step & Computational Time [s] & Nb of Operations & Complexity of Operations \\
\hline Step III (RCDPR Configuration Set) & 0.019 & $\prod_{v=1}^{n_{v}} \epsilon_{v}$ & Low \\
Step VI (Feasibility Map) & 142335 & $\prod_{v=1}^{n_{v}} \epsilon_{v} n_{p}$ & High \\
Step VII (Configuration Selection) & 10.61 & Non Predictable & Medium \\
Step VIII (Graph Building) & 492.64 & $n_{p} n_{\phi}$ & High \\
Step X (Dijkstra's Algorithm) & 0.018 & Non Predictable & Low \\
\hline
\end{tabular}




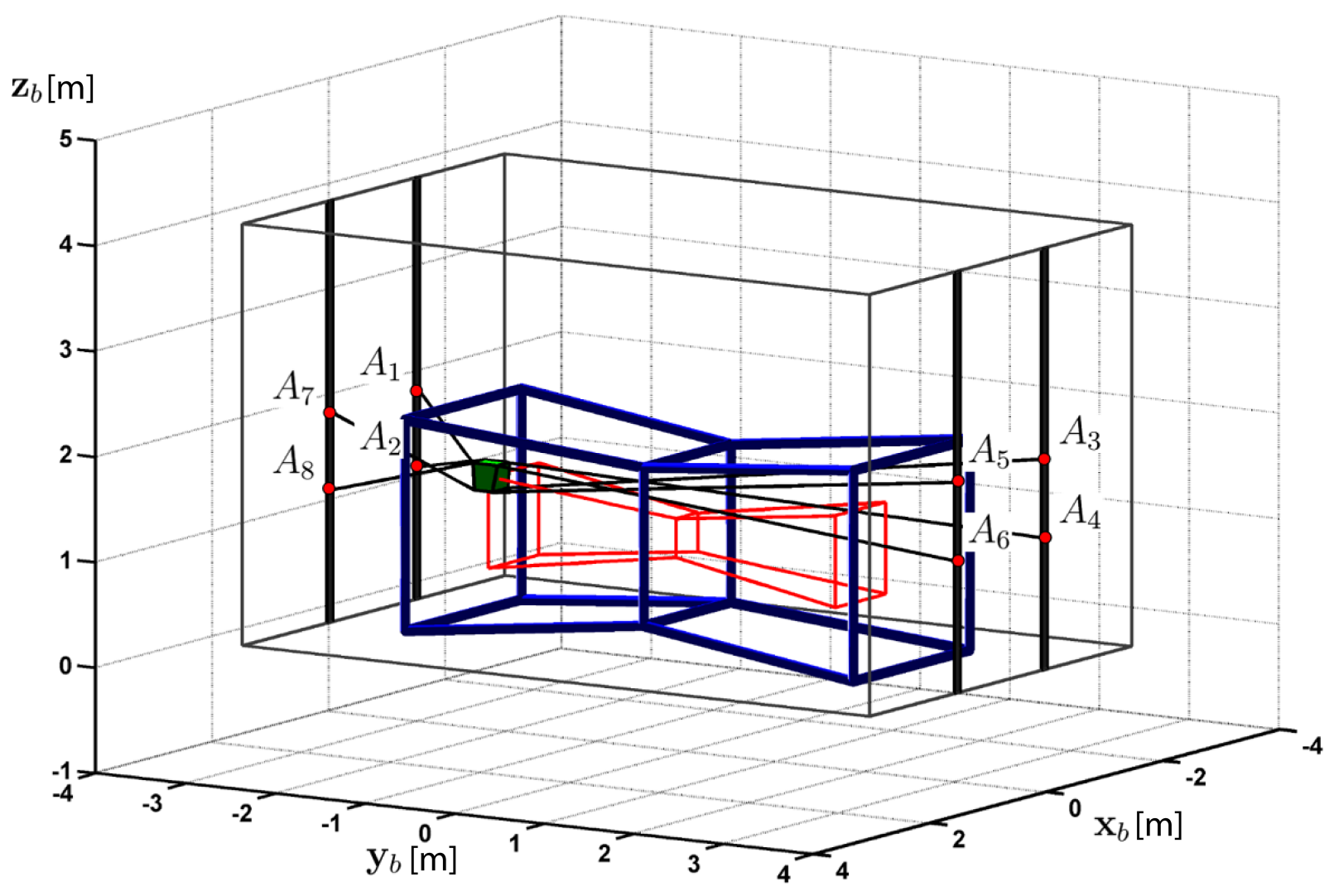

Figure 21: RCDPR configuration, with $w_{1}=0.75 \mathrm{~m}, w_{2}=2 \mathrm{~m}$ and $w_{3}=1.25 \mathrm{~m}$, which optimizes $\mu_{4}$ and $\mu_{5}$ for the inner prescribed path $\mathscr{P}_{\text {in }}$ 


\section{Conclusions}

This paper dealt with RCDPRs discrete reconfiguration planning. The considered task consists of low force operations, such as sandblasting and painting, in cluttered industrial environments and the RCDPR reconfigurability results from movable cable exit points. These exit points can be positioned at a possibly large but discrete set of possible locations. Means to select and optimize the sequence of discrete reconfigurations allowing the RCDPR moving platform to follow a prescribed path were introduced. A so-called feasibility map was first defined and generated. This map reflects the feasibility of all possible RCDPR configurations at all points of the discretized prescribed path, with respect to a set of constraints. In this paper, the latter set included wrench-feasibility, cable collision, and pose error constraints. Then, the feasibility map is analyzed and filtered in order to determine minimum sets of configurations which allow the RCDPR to follow the whole prescribed path. Based on these minimum sets, a graph-based approach was proposed to solve the discrete reconfiguration planning problem by providing a reconfiguration sequence which is optimal with respect to one of several possible user-defined cost functions. A case study which consists in following a path covering the whole inner and outer parts a tubular structure was finally presented. The results notably show that an optimal solution, which minimizes the number of required RCDPR reconfigurations, can be found.

The proposed discrete reconfiguration planning method may require further improvements, notably in order to reduce the computational time of the constraint analysis (feasibility map). As part of our future works, heuristic approaches will be considered. Indeed, with such approaches, it is presumably possible to obtain solutions to the discrete reconfiguration problem without analyzing all the constraints of all the possible configurations. Such solutions would not be optimal as the ones considered in this paper but should be much faster to compute.

\section{Acknowledgments}

This research work is part of the CAROCA project managed by IRT Jules Verne (French Institute in Research and Technology in Advanced Manufacturing Technologies for Composite, Metallic and Hybrid Structures). The authors wish to associate the industrial and academic partners of this project, namely, STX, DCNS, AIRBUS and CNRS.

\section{References}

[1] L. Gagliardini, S. Caro, M. Gouttefarde, P. Wenger, A. Girin, A reconfigurable cable-driven parallel robot for sandblasting and painting of large structures, in: Cable-Driven Parallel Robots, Mechanism and Machine Science, Vol. 32, 2015, pp. 275-291.

[2] J. Albus, R. Bostelman, N. Dagalakis, The NIST spider, a robot crane, J. of Research of the Nat. Inst. of Standards and Technology 97 (3) (1992) 373-385.

[3] C. Holland, D. Cannon, U.s. patent 6826452, cable array robot for material handling (March 2002).

[4] M. Hassan, A. Khajepour, Analysis of large-workspace cable-actuated manipulator for warehousing applications, in: ASME International Design Engineering Technical Conferences, 2006. 
[5] A. Pott, C. Meyer, A. Verl, Large-scale assembly of solar power plants with parallel cable robots, in: International Symposium on Robotics, 2010, pp. 1-6.

[6] P. Gallina, G. Rosati, A. Rossi, 3-dof wire driven planar haptic interface, Journal of Intelligent and Robotic Systems 32 (1) (2001) 23-36.

[7] G. Rosati, M. Andreolli, A. Biondi, P. Gallina, Performance of cable suspended robots for upper limb rehabilitation, in: IEEE 10th International Conference on Rehabilitation Robotics (ICORR), 2007, pp. 385-392.

[8] A. Fortin-Coté, P. Cardou, C. Gosselin, An admittance control scheme for haptic interfaces based on cable-driven parallel mechanisms, in: 2014 IEEE International Conference on Robotics and Automation (ICRA), 2014, pp. 819-925.

[9] R. Yao, X. Tang, J. Wang, P. Huang, Dimensional optimization design for the four-cable driven parallel manipulator in FAST, IEEE/ASME Transactions on Mechatronics 15 (6) (2010) 932-941.

[10] R. Yao, H. Li, X. Zhang, A modeling method of the cable driven parallel manipulator for FAST, in: Cable-Driven Parallel Robots, Mechanism and Machine Science, Vol. 12, Springer, 2013, pp. 87-102.

[11] J.-P. Merlet, Kinematics of the wire-driven parallel robot MARIONET using linear actuators, in: Proc. IEEE Int. Conf. on Robotics and Automation, Passadena, 2008, pp. 3857-3862.

[12] J.-P. Merlet, D. Daney, A portable, modular parallel wire crane for rescue operations, in: 2010 IEEE International Conference on Robotics and Automation (ICRA), 2010, pp. 2834-2939.

[13] M. Gouttefarde, J.-F. Collard, N. Riehl, C. Baradat, Geometry selection of a redundantly actuated cable-suspended parallel robot, IEEE Trans. on Robotics 31 (2) (2015) 501-510.

[14] D.-Q. Nguyen, M. Gouttefarde, O. Company, F. Pierrot, On the analysis of large-dimension reconfigurable suspended cable-driven parallel robots, in: IEEE International Conference on Robotics and Automation (ICRA), 2014, pp. 5728-5735.

[15] J. Fink, N. Michael, S. Kim, V. Kumar, Planning and control for cooperative manipulation and transportation with aerial robots, Int. J. Rob. Research 30 (3) (2010) 324-334.

[16] Q. Jiang, V. Kumar, The inverse kinematics of cooperative transport with multiple aerial robots, IEEE Trans. on Robotics 29 (1) (2013) $136-145$.

[17] M. Manubens, D. Devaurs, L. Ros, J. Cortés, Motion planning for 6-d manipulation with aerial towed-cable systems, in: Robotics: Science and Systems, Berlin, Germany, 2013.

[18] L. Gagliardini, S. Caro, M. Gouttefarde, P. Wenger, A. Girin, A reconfiguration strategy for reconfigurable cable-driven parallel robots, in: IEEE International Conference on Robotics and Automation (ICRA), 2015.

[19] R. Bostelman, A. Jacoff, F. Proctor, T. Kramer, A. Wavering, Cable-based reconfigurable machines for large scale manufacturing, in: Proc. of the 2000 Japan-USA Symposium on Flexible Automation, Ann Arbor, MI, 2000.

[20] J.-B. Izard, M. Gouttefarde, O. Tempier, C. Baradat, A reconfigurable robot for cable-driven parallel robotic research and industrial scenario proofing, in: Cable-Driven Parallel Robots, Vol. 12, 2013, pp. 135-148.

[21] G. Rosati, D. Zanotto, S. K. Agrawal, On the design of adaptive cable-driven systems, Journal of Mechanisms and Robotics 3.

[22] D. Zanotto, G. Rosati, S. Minto, A. Rossi, Sophia-3: A semiadaptive cable-driven rehabilitation device with a tilting working plane, IEEE Trans. on Robotics 30 (4) (2014) 974-979.

[23] A. Zhou, C. P. Tang, V. Krovi, Analysis framework for cooperating mobile cable robots, in: IEEE Int. Conf. on Robotics and Automation, RiverCentre, Saint Paul, MN, 2012, pp. 3128-3133.

[24] X. Zhou, S. Jun, V. Krovi, Tension distribution shaping via reconfigurable attachment in planar mobile cable robots, Robotica 32 (2014) $245-256$

[25] X. Zhou, S.-K. Jun, V. Krovi, Stiffness modulation exploiting configuration redundancy in mobile cable robots, in: Proc. IEEE Int. Conf. Robotics and Automation, Hong Kong, China, 2014, pp. 5934-5939.

[26] R. Roberts, T. Graham, T. Lippitt, On the inverse kinematics, statics, and fault tolerance of cable-suspended robots, J. of Robotic Systems 15 (10) (1998) 581-597.

[27] S. Behzadipour, A. Khajepour, Stiffness of cable-based parallel manipulators with application to stability analysis, J. Mechanical Design 128 
(2006) 303-310.

[28] H. A. Eiselt, M. Gendreau, G. Laporte, Arc routing problems, Part I: The Chinese postman problem, Operational Research 43 (2) (1995) $231-242$

[29] S. Bouchard, C. M. Gosselin, B. Moore, On the ability of a cable-driven robot to generate a prescribed set of wrenches, in: Proc. of the ASME 2008 Int. Design Eng. Technical Conf. \& Computers and Information in Eng. Conf., Brooklyn, New York, USA,, 2008, pp. 1-12.

[30] M. Gouttefarde, S. Krut, Characterization of parallel manipulator available wrench set facets, in: Advances in Robot Kinematics: Motion in Man and Machine, Piran, 2010, pp. 475-484.

[31] V. Lumelsky, On fast computation of distance between line segments, Information Processing Letters 21.

[32] A. Cruz Ruiz, S. Caro, P. Cardou, F. Guay, Arachnis : Analysis of robots actuated by cables with handy and neat interface software, in: Mechanisms and Machine Science Volume, Vol. 32, 2015, pp. 293-305.

[33] J. Siek, L.-Q. Lee, A. Lumsdaine, The Boost Graph Library: User Guide and Reference Manual, Addison Wesley, 2001.

[34] E. W. Dikstra, A note on two problems in connection with graphs, Numerische Mathematik, 1959.

[35] S. Caro, D. Chablat, Y. Hu, Algorithm for the actuation mode selection of the parallel manipulator NaVARo, in: Proceeding of the ASME 2014 International Design Engineering Technical Conference \& Computers and Information in Engineering Conference IDETC/CIE 2014, Buffalo, NY, USA, 2014. 